\title{
Rare A-Type, Spiro-Type, and Highly Oligomeric Proanthocyanidins from Pinus massoniana
}

Bin Zhou, ${ }^{1, \dagger}$ Yvette Alania,, ${ }^{3,4}$ Mariana C. Reis, ${ }^{3,4}$ James B. McAlpine, ${ }^{1,2}$ Ana K. Bedran-Russo, ${ }^{2,3,4}$ Guido F. Pauli, ${ }^{1,2}$ and Shao-Nong Chen ${ }^{1,2, *}$

${ }^{1}$ Department of Pharmaceutical Sciences and ${ }^{2}$ Program for Collaborative Research in the Pharmaceutical Sciences (PCRPS), College of Pharmacy, ${ }^{3}$ Department of Restorative Dentistry, College of Dentistry, University of Illinois at Chicago, Chicago, Illinois 60612, USA; ${ }^{4}$ Department of General Dental Sciences, School of Dentistry, Marquette University, Milwaukee, Wisconsin 53233, USA.

'Present Address: State Key Laboratory of Drug Research, Shanghai Institute of Materia Medica, Chinese Academy of Sciences, 555 Zuchongzhi Road, Shanghai 201203, People's Republic of China 


\section{Table of Contents}

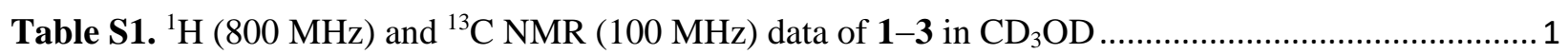

Figure S1. (A) Selected COSY (bold bonds), HMBC, and (B) NOESY correlations (arrows) in 1 ............ 3

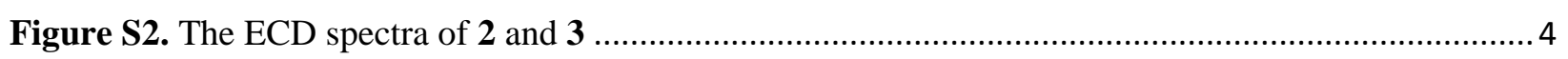

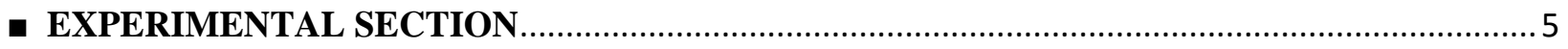

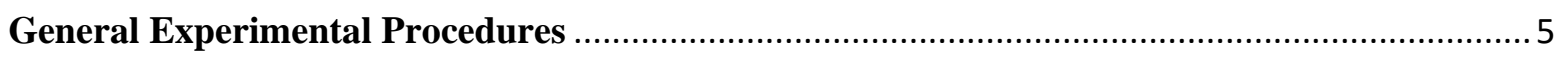

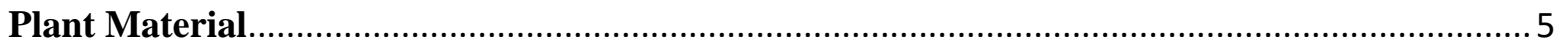

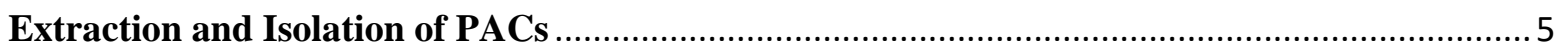

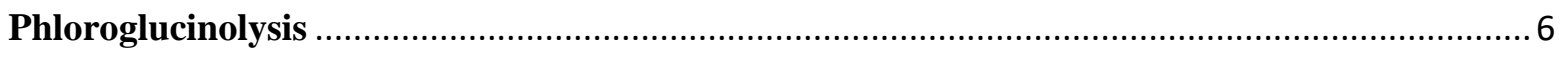

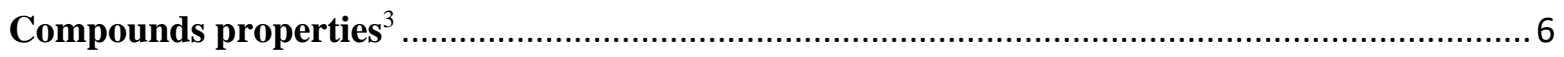

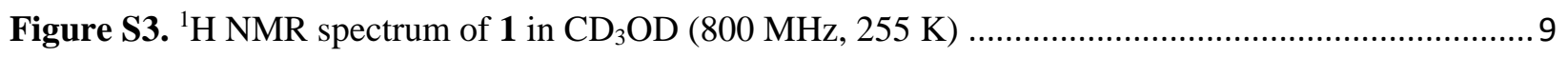

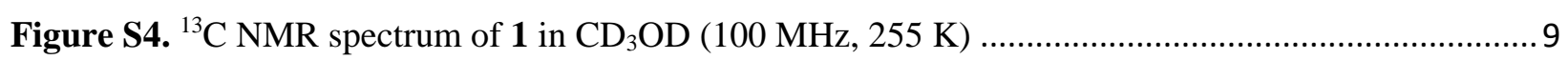

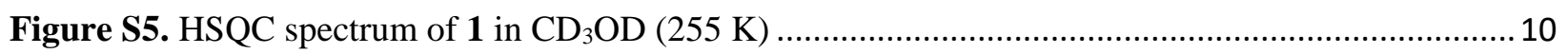

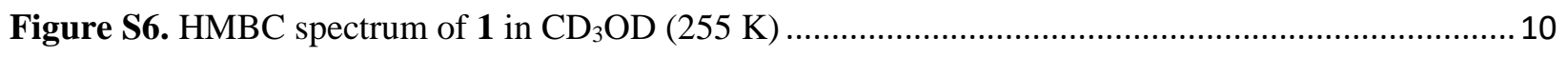

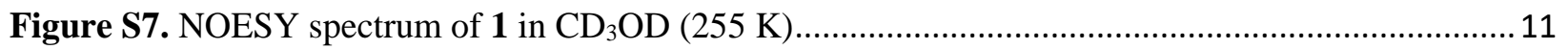

Figure S7-1. Partially expanded NOESY spectra of 1 with important NOESY correlations ...................11

Figure S7-2. Expanded NOESY spectra of 1 with important NOESY correlations ................................ 12

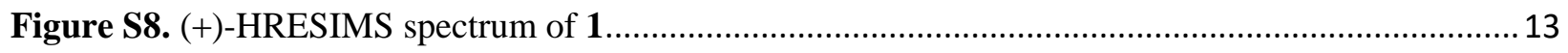

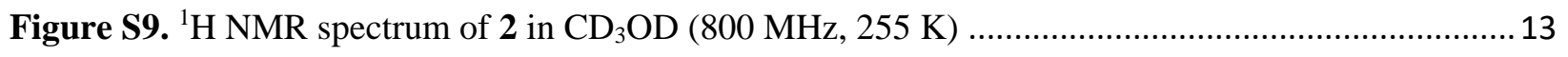

Figure S10. Comparison of the ${ }^{1} \mathrm{H}$ NMR spectra of 2 in $\mathrm{CD}_{3} \mathrm{OD}$ at $278 \mathrm{~K}$ and $255 \mathrm{~K}(800 \mathrm{MHz}) \ldots \ldots \ldots . . .14$

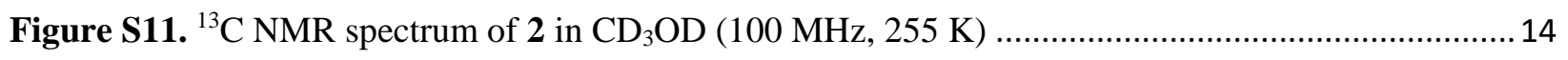

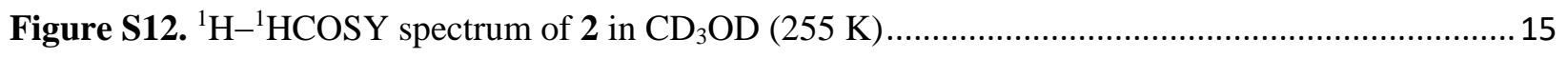

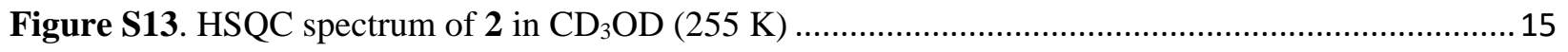

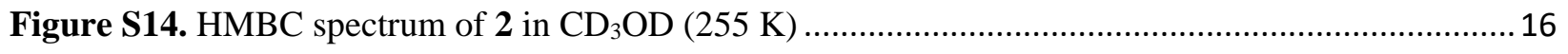

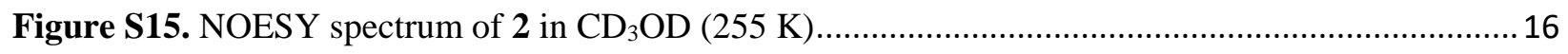

Figure S15-1. Partially expanded NOESY spectra of 2 with important NOESY correlations ................. 17

Figure S15-2. Expanded NOESY spectra of 2 with important NOESY correlations ............................. 17

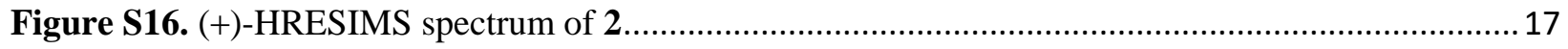




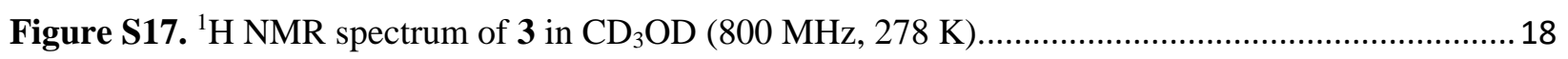

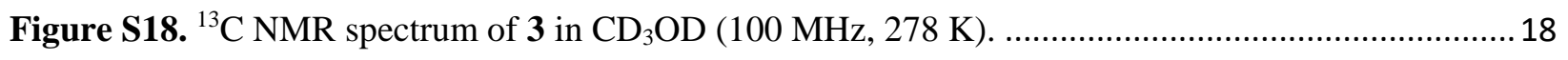

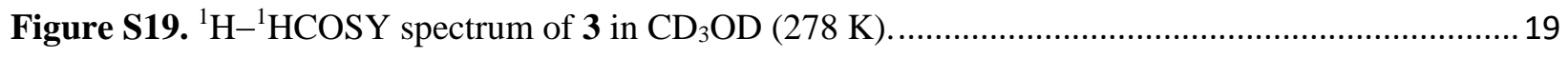

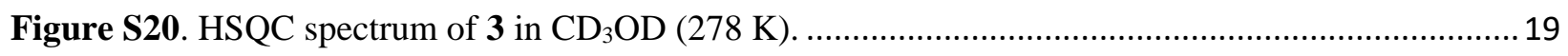

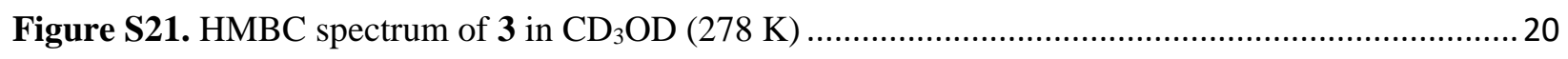

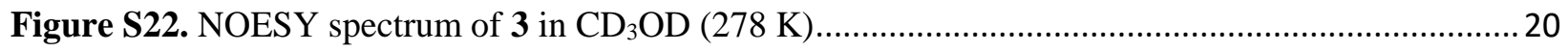

Figure S22-1. Partially expanded NOESY spectra of $\mathbf{3}$ with important NOESY correlations ................. 21

Figure S22-2. Expanded NOESY spectra of $\mathbf{3}$ with important NOESY correlations .............................. 21

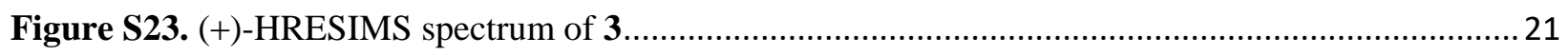

Figure S24. Phloroglucinolysis products of $\mathbf{3}$ identified by chiral phase HPLC and MS analysis ............22

Figure S25. Calculation of the purity of $\mathbf{1}$ by qHNMR using the $100 \%$ method ....................................23

Figure S26. Calculation of the purity of $\mathbf{2}$ by qHNMR using the $100 \%$ method .....................................24

Figure S27. Calculation of the purity of $\mathbf{3}$ by qHNMR using the $100 \%$ method ....................................25 
Table S1. ${ }^{1} \mathrm{H}(800 \mathrm{MHz})$ and ${ }^{13} \mathrm{C}$ NMR $(100 \mathrm{MHz})$ data of $\mathbf{1}-\mathbf{3}$ in $\mathrm{CD}_{3} \mathrm{OD}$

\begin{tabular}{|c|c|c|c|c|c|c|c|c|c|c|c|c|c|}
\hline \multirow{2}{*}{ Unit } & \multirow{2}{*}{ Ring } & \multirow{2}{*}{ No. } & \multicolumn{2}{|l|}{$\mathbf{1}^{a}$} & \multicolumn{2}{|l|}{$2^{a}$} & \multicolumn{2}{|l|}{$3^{b}$} & \multirow{2}{*}{ Unit } & \multirow{2}{*}{ Ring } & \multirow{2}{*}{ No. } & \multicolumn{2}{|l|}{$3^{b}$} \\
\hline & & & $\delta_{\mathrm{H}}$, mult $(J$ in $\mathrm{Hz})$ & $\delta_{\mathrm{C}}$ & $\delta_{\mathrm{H}}$, mult $(J$ in $\mathrm{Hz})$ & $\delta \mathrm{C}$ & $\delta_{\mathrm{H}}$, mult $(J$ in $\mathrm{Hz})$ & $\delta \mathrm{C}$ & & & & $\delta_{\mathrm{H}}$, mult $(\mathrm{J}$ in $\mathrm{Hz})$ & $\delta \mathrm{C}$ \\
\hline \multirow[t]{15}{*}{ I } & $\mathrm{C}$ & 2 & & 100.5 & & 99.9 & & 100.0 & $\mathrm{~V}$ & $\mathrm{O}$ & 2 & & 100.2 \\
\hline & & 3 & $3.867, \mathrm{~d}(4.0)$ & 65.1 & $3.690, \mathrm{~d}(3.5)$ & 67.5 & $4.129, \mathrm{~d}(3.4)$ & 68.2 & & & 3 & 4.117, d (3.6) & 67.6 \\
\hline & & 4 & $4.258, \mathrm{~d}(4.0)$ & 29.7 & $4.437, \mathrm{~d}(3.5)$ & 28.9 & $4.468, \mathrm{~d}(3.4)$ & 29.4 & & & 4 & $4.227, \mathrm{~d}(3.6)$ & 29.6 \\
\hline & A & 5 & & 155.2 & & 156.9 & & 157.1 & & M & 5 & & 154.1 \\
\hline & & 6 & $5.922, \mathrm{~d}(1.9)$ & 97.3 & $6.032, \mathrm{~d}(2.3)$ & 98.0 & $6.057, \mathrm{~d}(2.4)$ & 98.2 & & & 6 & & 111.0 \\
\hline & & 7 & & 157.9 & & 157.9 & & 158.0 & & & 7 & & 156.7 \\
\hline & & 8 & $5.867, \mathrm{~d}(1.9)$ & 96.1 & $6.023, \mathrm{~d}(2.3)$ & 96.2 & $6.111, \mathrm{~d}(2.4)$ & 96.6 & & & 8 & $6.076, \mathrm{~s}$ & 97.5 \\
\hline & & 9 & & 154.8 & & 154.1 & & 154.1 & & & 9 & & 152.1 \\
\hline & & 10 & & 105.3 & & 104.4 & & 104.2 & & & 10 & & 104.0 \\
\hline & B & $1^{\prime}$ & & 131.7 & & 131.9 & & 132.5 & & $\mathrm{~N}$ & $1^{\prime}$ & & 132.0 \\
\hline & & $2^{\prime}$ & $7.274, \mathrm{~d}(2.1)$ & 116.1 & $7.011, \mathrm{~d}(2.1)$ & 115.2 & $7.179, \mathrm{~d}(2.1)$ & 115.5 & & & $2^{\prime}$ & $7.139, \mathrm{~d}(2.2)$ & 115.5 \\
\hline & & $3^{\prime}$ & & 145.2 & & 145.0 & & 145.5 & & & $3^{\prime}$ & & 145.6 \\
\hline & & $4^{\prime}$ & & 146.6 & & 146.2 & & 146.6 & & & $4^{\prime}$ & & 146.7 \\
\hline & & $5^{\prime}$ & $6.764, \mathrm{~d}(8.2)$ & 115.1 & $6.713, \mathrm{~d}(8.2)$ & 115.5 & $6.837, \mathrm{~d}(8.2)$ & 115.5 & & & $5^{\prime}$ & $6.815, \mathrm{~d}(8.3)$ & 115.5 \\
\hline & & $6^{\prime}$ & $6.904, \mathrm{dd}(8.2,2.1)$ & 120.3 & $6.607, \mathrm{dd}(8.2,2.1)$ & 119.4 & $7.060, \mathrm{dd}(8.2,2.1)$ & 119.7 & & & $6^{\prime}$ & $7.041, \mathrm{dd}(8.3,2.2)$ & 119.8 \\
\hline \multirow[t]{15}{*}{ II } & $\mathrm{F}$ & 2 & & 101.1 & 5.301, brs & 78.7 & $5.268, \mathrm{~s}$ & 78.7 & VI & $\mathrm{R}$ & 2 & 4.781, d (7.7) & 85.1 \\
\hline & & 3 & $4.216, \mathrm{~d}(4.3)$ & 68.7 & 3.944 , brs & 72.3 & 4.043 , brs & 72.3 & & & 3 & $4.192, \mathrm{~m}$ & 68.2 \\
\hline & & 4 & $4.866, \mathrm{~d}(4.3)$ & 30.4 & 4.472 , brs & 38.1 & 4.739, brs & 38.6 & & & 4 & $2.607, \mathrm{dd}(-16.2,8.4)$ & 29.0 \\
\hline & $\mathrm{D}$ & 5 & & 150.8 & & 157.1 & & 156.4 & & $\mathrm{P}$ & 4 & $2.996, \mathrm{dd}(-16.2,5.1)$ & 29.0 \\
\hline & & 6 & & 108.5 & $5.960, \mathrm{~s}$ & 95.8 & $6.126, \mathrm{~s}$ & 96.1 & & & 5 & & 156.2 \\
\hline & & 7 & & 153.1 & & 151.9 & & 152.2 & & & 6 & $6.136, \mathrm{~s}$ & 96.6 \\
\hline & & 8 & $6.254, \mathrm{~s}$ & 97.4 & & 106.3 & & 106.8 & & & 7 & & 152.1 \\
\hline & & 9 & & 154.6 & & 152.7 & & 152.3 & & & 8 & & 106.4 \\
\hline & & 10 & & 105.9 & & 105.0 & & 105.6 & & & 9 & & 151.2 \\
\hline & $\mathrm{E}$ & $1^{\prime}$ & & 131.5 & & 131.5 & & 131.4 & & Q & 10 & & 103.1 \\
\hline & & $2^{\prime}$ & $6.982, \mathrm{~d}(2.2)$ & 115.4 & $7.210, \mathrm{~d}(2.1)$ & 116.6 & $7.157, \mathrm{~d}(2.1)$ & 116.2 & & & $1^{\prime}$ & & 130.0 \\
\hline & & $3^{\prime}$ & & 145.5 & & 145.8 & & 145.8 & & & $2^{\prime}$ & $6.997, \mathrm{~d}(2.1)$ & 115.9 \\
\hline & & $4^{\prime}$ & & 146.6 & & 146.3 & & 146.2 & & & $3^{\prime}$ & & 146.2 \\
\hline & & $5^{\prime}$ & $6.745, \mathrm{~d}(8.1)$ & 115.5 & $6.799, \mathrm{~d}(8.2)$ & 115.6 & $6.796, \mathrm{~d}(8.2)$ & 115.9 & & & $4^{\prime}$ & & 146.9 \\
\hline & & $6^{\prime}$ & $6.916, \mathrm{dd}(8.1,2.2)$ & 119.7 & $7.075, \mathrm{dd}(8.2,2.1)$ & 121.4 & $6.962, \mathrm{dd}(8.2,2.1)$ & 120.7 & & & $5^{\prime}$ & $6.878, \mathrm{~d}(8.1)$ & 116.8 \\
\hline \multirow[t]{2}{*}{ III } & I & 2 & $4.964, \mathrm{~s}$ & 79.7 & $5.810, \mathrm{~s}$ & 88.2 & & 100.1 & & & $6^{\prime}$ & $6.924, \mathrm{dd}(8.1,2.1)$ & 121.1 \\
\hline & & 3 & $4.279, \mathrm{~m}$ & 66.9 & & 61.4 & $4.167, \mathrm{~d}(3.6)$ & 68.0 & & & & & \\
\hline
\end{tabular}




\begin{tabular}{|c|c|c|c|c|c|c|c|c|}
\hline & & 4 & $\begin{array}{c}2.025, \mathrm{dd}(-17.0,3.5) \\
2.596, \operatorname{brd}(-17.0)\end{array}$ & $\begin{array}{l}23.6 \\
23.6\end{array}$ & & 177.0 & 4.383, d (3.6) & 29.8 \\
\hline & G & 5 & & 156.4 & & 154.2 & & 154.6 \\
\hline & & 6 & $5.952, \mathrm{~s}$ & 95.7 & $5.902, \mathrm{~s}$ & 96.6 & & 110.5 \\
\hline & & 7 & & 151.7 & & 158.2 & & 156.8 \\
\hline & & 8 & & 105.6 & & 106.0 & $6.076, \mathrm{~s}$ & 97.4 \\
\hline & & 9 & & 152.4 & & 162.2 & & 152.1 \\
\hline & & 10 & & 101.1 & & 106.1 & & 104.4 \\
\hline & $\mathrm{H}$ & $1^{\prime}$ & & 132.4 & & 128.5 & & 132.3 \\
\hline & & $2^{\prime}$ & $6.437, \mathrm{~d}(2.2)$ & 113.1 & $6.411, \mathrm{~d}(2.2)$ & 113.3 & $7.163, \mathrm{~d}(2.2)$ & 115.5 \\
\hline & & $3^{\prime}$ & & 146.1 & & 145.7 & & 145.6 \\
\hline & & $4^{\prime}$ & & 145.5 & & 145.8 & & 146.7 \\
\hline & & $5^{\prime}$ & $6.432, \mathrm{~d}(8.1)$ & 115.5 & $6.629, \mathrm{~d}(8.3)$ & 115.9 & $6.827, \mathrm{~d}(8.3)$ & 115.5 \\
\hline & & 6 & $6.038, \mathrm{dd}(8.1,2.2)$ & 117.6 & $6.532, \mathrm{dd}(8.3,2.2)$ & 118.3 & $7.055, \mathrm{dd}(8.3,2.2)$ & 119.7 \\
\hline IV & $\mathrm{L}$ & 2 & & & $4.830, \mathrm{~d}(6.3)$ & 82.5 & $5.415, \mathrm{~s}$ & 79.9 \\
\hline & & 3 & & & $3.973, \mathrm{~m}$ & 68.7 & 3.866 , brs & 72.6 \\
\hline & & 4 & & & $2.676, \mathrm{dd}(-16.4,7.2)$ & 27.6 & 4.787, brs & 38.4 \\
\hline & $\mathrm{J}$ & & & & $2.857, \mathrm{dd}(-16.4,5.0)$ & 27.6 & & \\
\hline & & 5 & & & & 158.1 & & 156.7 \\
\hline & & 6 & & & $6.163, \mathrm{~s}$ & 91.3 & $6.109, \mathrm{~s}$ & 96.6 \\
\hline & & 7 & & & & 154.5 & & 151.9 \\
\hline & & 8 & & & & 104.0 & & 106.2 \\
\hline & & 9 & & & & 152.8 & & 152.6 \\
\hline & $\mathrm{K}$ & 10 & & & & 104.8 & & 106.0 \\
\hline & & $1^{\prime}$ & & & & 131.64 & & 130.8 \\
\hline & & $2^{\prime}$ & & & $6.795, \mathrm{~d}(2.1)$ & 114.0 & $7.204, \mathrm{~d}(2.1)$ & 117.1 \\
\hline & & $3^{\prime}$ & & & & 145.9 & & 145.5 \\
\hline & & $4^{\prime}$ & & & & 146.0 & & 146.5 \\
\hline & & $5^{\prime}$ & & & $6.744, d(8.1)$ & 115.8 & $6.778, d(8.1)$ & 115.9 \\
\hline & & $6^{\prime}$ & & & $6.677, \mathrm{dd}(8.1,2.1)$ & 119.7 & $6.935, \mathrm{dd}(8.1,2.1)$ & 121.8 \\
\hline
\end{tabular}

Data were acquired at ${ }^{a} 255 \mathrm{~K}$ and ${ }^{b} 278 \mathrm{~K}$. 
Figure S1. (A) Selected COSY (bold bonds), HMBC, and (B) NOESY correlations (arrows) in 1

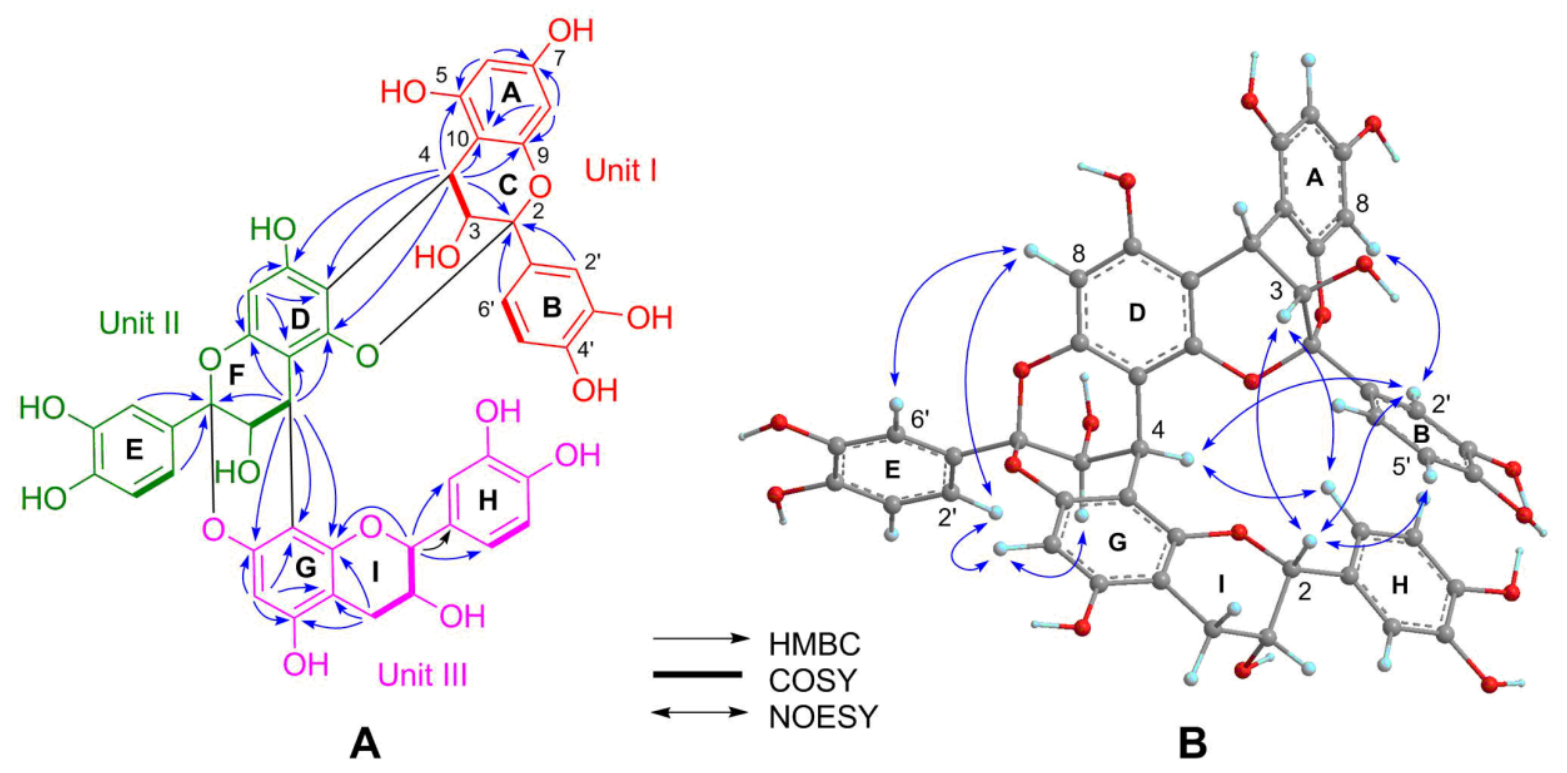


Figure S2. The ECD spectra of $\mathbf{2}$ and $\mathbf{3}$
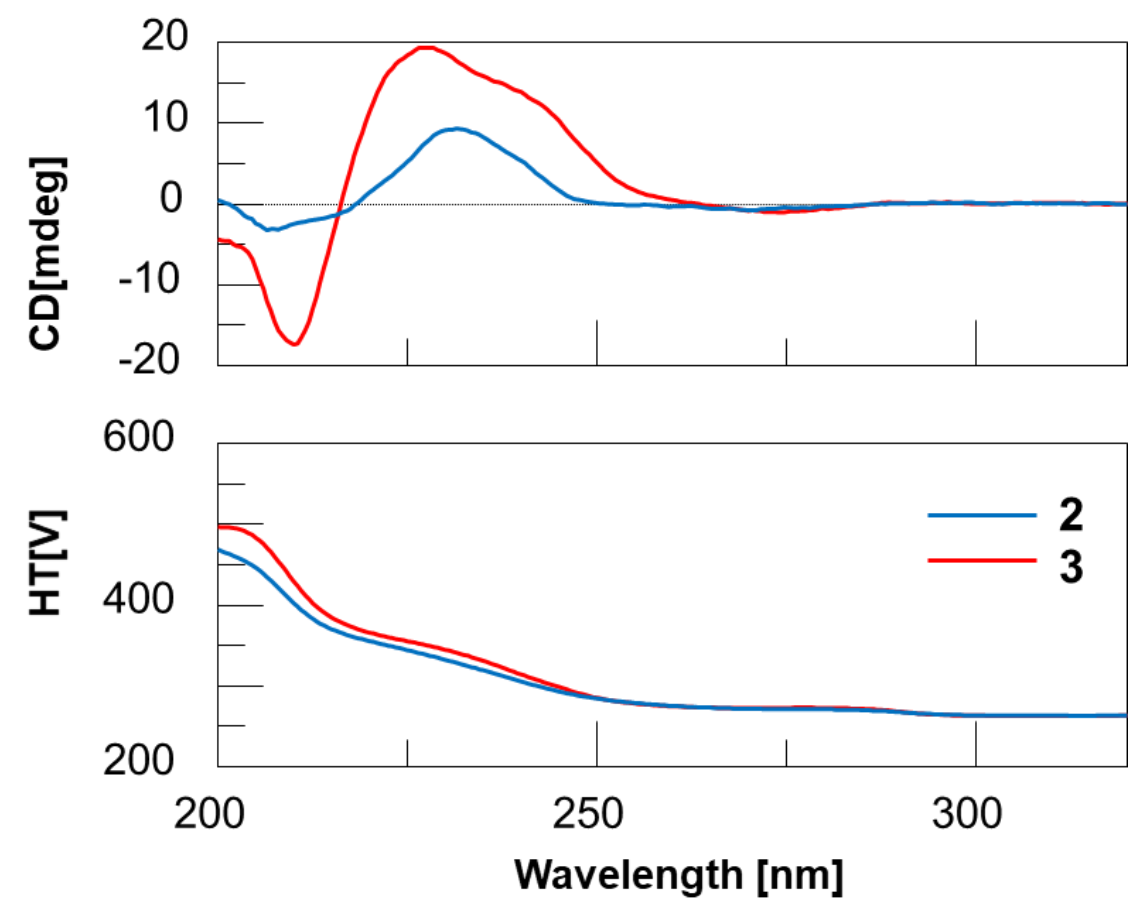


\section{- EXPERIMENTAL SECTION}

\section{General Experimental Procedures}

HRESIMS measurements were carried out using a Bruker (Billerica, MA, USA) Impact II quadrupole time-of-flight (qTOF-) spectrometer. The ECD spectra were acquired on a JASCO715 spectrometer. Samples for ECD experiments were prepared in $\mathrm{MeOH}$ at $\leq 0.1 \mathrm{mg} / \mathrm{mL}$, and a $0.2 \mathrm{~cm}$ path length quartz cuvette was used. All ${ }^{1} \mathrm{H}$ 1D/2D NMR spectra were acquired at $255 \mathrm{~K}$ or $278 \mathrm{~K}$ on an $800 \mathrm{MHz}$ Bruker (Billerica, MA, USA) Avance spectrometer equipped with a 5 mm triple resonance inverse TCI RT probe. The ${ }^{13} \mathrm{C}$ NMR experiments of all compounds were performed on a JEOL (JEOL Resonance, Peabody, MA, USA) ECZ 400 MHz spectrometer. Silica gel (230-400 mesh) was used for column chromatography. $\mathrm{C}_{18}$ reversed-phase (RP-18) silica gel (20-45 $\mu \mathrm{m}$, MACHEREY-NAGEL, Bethlehem, PA, USA) and Sephadex LH-20 gel (Amersham Biosciences) were used for column chromatography. Pre-coated silica gel GF254 plates (MACHEREY-NAGEL, Bethlehem, PA, USA) were used for TLC monitoring. Semi-preparative HPLC was performed on a Shimadzu HPLC (Kyoto, Japan) connected to a PDA detector (Shimadzu, model SPD-20A) and equipped with a YMC-Pack ODS-AQ $(250 \times 10 \mathrm{~mm}, S-5 \mu \mathrm{m}$, $120 \AA$ A YMC America, Allentown, USA) or Chiralpak IA $(250 \times 10.0 \mathrm{~mm}, S-5 \mu \mathrm{m}$; Chiral Technologies, West Chester, PA, USA) column. All solvents were of analytical grade from Fisher Scientific (Hanover Park, IL, USA) or Sigma-Aldrich (St. Louis, MO, USA).

\section{Plant Material}

Extract powder of the inner bark of Pinus massoniana was purchased from Xi'an Chukang Biotechnology in China in 2012 (No. PB120212).

\section{Extraction and Isolation of PACs}

$12 \mathrm{~g}$ of enriched tri- and tetrameric proanthocyanidins, separated into $6.5 \mathrm{~g}$ of fraction A and 5.6 $\mathrm{g}$ of fraction $\mathrm{B}$, were prepared from $200 \mathrm{~g}$ pine bark extract by centrifugal partition chromatography (CPC) as described previously. ${ }^{1}$ Both fractions A and B were chromatographed on a Sephadex LH-20 column (ethanol), affording six (A1-A6) and seven subfractions (B1-B7), respectively. Fraction B5 (800 mg) was separated over a RP-18 silica gel column $\left(\mathrm{MeOH} / \mathrm{H}_{2} \mathrm{O}\right.$, 20-80\%), and the two major subfractions B5b and B5c were then purified via semi-preparative HPLC (18\% ACN in $0.1 \%$ formic acid $\left.\mathrm{H}_{2} \mathrm{O}, 2.5 \mathrm{~mL} / \mathrm{min}\right)$ to afford $1(0.7 \mathrm{mg})$ and $2(0.8 \mathrm{mg})$. 
Compound 3 (20 mg) was purified from fraction B7 by semi-preparative HPLC (20\% ACN in $0.1 \%$ formic acid $\mathrm{H}_{2} \mathrm{O}, 2.5 \mathrm{~mL} / \mathrm{min}$ ) after enrichment via a RP-18 silica gel column.

\section{Phloroglucinolysis}

Phloroglucinolysis was performed as described previously ${ }^{2}$ to corroborate the absolute configuration of $\mathbf{3}$. The identity of the reaction products was confirmed by chiral phase HPLC and MS analysis (Figure S24).

\section{Compounds properties ${ }^{3}$}

Pinutwindoublin (1): ent-epicatechin- $(2 \alpha \rightarrow \mathrm{O} \rightarrow 5,4 \alpha \rightarrow 6)$-ent-epicatechin- $(2 \alpha \rightarrow \mathrm{O} \rightarrow 7,4 \alpha \rightarrow 8)$ epicatechin: light brown, amorphous solid; ECD $(\mathrm{MeOH}) \lambda_{\max }(\Delta \varepsilon) 211(+21.6), 232(-20.8), 287$ $(-0.8) \mathrm{nm} ;{ }^{1} \mathrm{H}$ NMR (Methanol-d4, $\left.800 \mathrm{MHz}, 255 \mathrm{~K}\right): \delta 2.025(\mathrm{dd}, 1 \mathrm{H}, J=17.0,3.5 \mathrm{~Hz}), 2.596$ (brd, $1 \mathrm{H}, J=17.0 \mathrm{~Hz}), 3.867(\mathrm{~d}, 1 \mathrm{H}, J=4.0 \mathrm{~Hz}), 4.216(\mathrm{~d}, 1 \mathrm{H}, J=4.3 \mathrm{~Hz}), 4.258(\mathrm{~d}, 1 \mathrm{H}, J=4.0$ Hz), 4.279 (m, 1H), 4.866 (d, 1H, J=4.3 Hz), 4.964 (s, 1H), 5.867 (d, 1H, J = 1.9 Hz), 5.922 (d, $1 \mathrm{H}, J=1.9 \mathrm{~Hz}), 5.952(\mathrm{~s}, 1 \mathrm{H}), 6.038(\mathrm{~d}, 1 \mathrm{H}, J=8.3 \mathrm{~Hz}), 6.254(\mathrm{~s}, 1 \mathrm{H}), 6.432$ (d, 1H, $J=8.1 \mathrm{~Hz})$, $6.437(\mathrm{~d}, 1 \mathrm{H}, J=2.2 \mathrm{~Hz}), 6.745(\mathrm{~d}, 1 \mathrm{H}, J=8.1 \mathrm{~Hz}), 6.764(\mathrm{~d}, 1 \mathrm{H}, J=8.2 \mathrm{~Hz}), 6.904(\mathrm{dd}, 1 \mathrm{H}, J=$ 8.3, 2.1 Hz), $6.916(\mathrm{dd}, 1 \mathrm{H}, J=8.1,2.2 \mathrm{~Hz}), 6.982(\mathrm{~d}, 1 \mathrm{H}, J=2.1 \mathrm{~Hz}), 7.274(\mathrm{~d}, 1 \mathrm{H}, J=2.1 \mathrm{~Hz})$. ${ }^{13} \mathrm{C}\left\{{ }^{1} \mathrm{H}\right\}$ NMR (Methanol-d4, $100 \mathrm{MHz}, 255 \mathrm{~K}$ ): $\delta$ 23.6, 29.7, 30.4, 65.1, 66.9, 68.7, 79.7, 95.7, 96.1, 97.3, 97.4, 100.5, 101.1, 101.1, 105.3, 105.6, 105.9, 108.5, 113.1, 115.1, 115.4, 115.5, 115.5, 116.1, 117.6, 119.7, 120.3, 131.5, 131.7, 132.4, 145.2, 145.5, 145.5, 146.1, 146.6, 146.6, 150.8, 151.7, 152.4, 153.1, 154.6, 154.8, 155.2, 156.4, 157.9; HRMS (ESI) $m / z[\mathrm{M}+\mathrm{H}]^{+}$Calcd for $\mathrm{C}_{45} \mathrm{H}_{35} \mathrm{O}_{18}$ 863.1818; Found 863.1823.

Pinuspirotetrin (2): Light brown, amorphous solid; ECD (MeOH) $\lambda_{\max }(\Delta \varepsilon) 207$ (-3.2), $232(+9.4)$, 270 (-0.8); ${ }^{1} \mathrm{H}$ NMR (Methanol-d4, $\left.800 \mathrm{MHz}, 255 \mathrm{~K}\right): \delta 2.676(\mathrm{dd}, 1 \mathrm{H}, J=16.4,7.2 \mathrm{~Hz}), 2.857$ $(\mathrm{dd}, 1 \mathrm{H}, J=16.4,5.0 \mathrm{~Hz}), 3.690(\mathrm{~d}, 1 \mathrm{H}, J=3.5 \mathrm{~Hz}), 3.944(\mathrm{brs} 1 \mathrm{H}), 3.973(\mathrm{~m}, 1 \mathrm{H}), 4.437$ (d, 1H, $J=3.5 \mathrm{~Hz}$ ), 4.472 (brs, 1H), 4.830 (d, 1H, $J=6.3 \mathrm{~Hz}), 5.301$ (brs, 1H), 5.810 (s, 1H), 5.902 (s, 1H), 5.960 (s, 1H), $6.023(\mathrm{~d}, 1 \mathrm{H}, J=2.3 \mathrm{~Hz}), 6.032$ (d, 1H, $J=2.5 \mathrm{~Hz}), 6.163(\mathrm{~s}, 1 \mathrm{H}), 6.629$ (d, $1 \mathrm{H}, J=8.3 \mathrm{~Hz}), 6.411(\mathrm{~d}, 1 \mathrm{H}, J=2.2 \mathrm{~Hz}), 6.532(\mathrm{dd}, 1 \mathrm{H}, J=8.1,2.3 \mathrm{~Hz}), 6.607(\mathrm{dd}, 1 \mathrm{H}, J=8.4$, $2.1 \mathrm{~Hz}), 6.677(\mathrm{dd}, 1 \mathrm{H}, J=8.2,2.1 \mathrm{~Hz}), 6.713(\mathrm{~d}, 1 \mathrm{H}, J=8.2 \mathrm{~Hz}), 6.744(\mathrm{~d}, 1 \mathrm{H}, J=8.1 \mathrm{~Hz}), 6.794$ $(\mathrm{d}, 1 \mathrm{H}, J=2.1 \mathrm{~Hz}), 6.799(\mathrm{~d}, 1 \mathrm{H}, J=8.2 \mathrm{~Hz}), 7.011(\mathrm{~d}, 1 \mathrm{H}, J=2.1 \mathrm{~Hz}), 7.075(\mathrm{dd}, 1 \mathrm{H}, J=8.2$, $2.1 \mathrm{~Hz}), 7.210(\mathrm{~d}, 1 \mathrm{H}, J=2.1 \mathrm{~Hz}) .{ }^{13} \mathrm{C}\left\{{ }^{1} \mathrm{H}\right\} \mathrm{NMR}$ (Methanol-d4, $100 \mathrm{MHz}, 255 \mathrm{~K}$ ): $\delta$ 27.6, 28.9, 
$38.1,61.4,67.5,68.7,72.3,78.7,82.5,88.2,91.3,95.8,96.2,96.6,98.0,99.9,104.0,104.4,104.8$, 105.0, 106.0, 106.1, 106.3, 113.3, 114.0, 115.2, 115.5, 115.6, 115.8, 115.9, 116.6, 118.3, 119.4, 119.7, 121.4, 128.5, 131.5, 131.6, 131.9, 145.0, 145.7, 145.8, 145.8, 145.9, 146.0, 146.2, 146.3, $151.9,152.7,152.8,154.1,154.2,154.5,156.9,157.1,157.9,158.1,158.2,162.2,177.0$; HRMS (ESI) $m / z[\mathrm{M}+\mathrm{H}]^{+}$Calcd for $\mathrm{C}_{60} \mathrm{H}_{45} \mathrm{O}_{24}, 1149.2295$; Found 1149.2291 .

Pinumassohexin (3): $\quad$ epicatechin- $(2 \beta \rightarrow \mathrm{O} \rightarrow 7,4 \beta \rightarrow 8)$-epicatechin-( $4 \beta \rightarrow 6)$-epicatechin$(2 \beta \rightarrow \mathrm{O} \rightarrow 7,4 \beta \rightarrow 8)$-epicatechin- $(2 \beta \rightarrow \mathrm{O} \rightarrow 7,4 \beta \rightarrow 8)$-epicatechin-( $(\beta \rightarrow 6)$-catechin: light brown, amorphous solid; ECD $(\mathrm{MeOH}) \lambda_{\max }(\Delta \varepsilon) 210(-17.3), 228(+19.3), 274(-1.0) ;{ }^{1} \mathrm{H}$ NMR (Methanol- $d_{4}, 800 \mathrm{MHz}, 278 \mathrm{~K}$ ): $\delta 2.607$ (dd, $1 \mathrm{H}, J=16.2,8.4 \mathrm{~Hz}$ ), 2.996 (dd, $1 \mathrm{H}, J=16.2,5.1$ Hz), 3.886 (brs, 1H), 4.043 (brs, 1H), 4.117 (d, 1H, $J=3.6 \mathrm{~Hz}$ ), 4.129 (d, 1H, $J=3.4$ Hz), 4.167 (d, $1 \mathrm{H}, J=3.5 \mathrm{~Hz}), 4.192(\mathrm{~m}, 1 \mathrm{H}), 4.227$ (d, 1H, $J=3.6 \mathrm{~Hz}), 4.383$ (d, 1H, $J=3.6 \mathrm{~Hz}), 4.468$ (d, $1 \mathrm{H}, J=3.4 \mathrm{~Hz}$ ), 4.739 (s, 1H), 4.781 (d, 1H $J=7.7), 4.787$ (s, 1H), 5.268 (s, 1H), 5.415 (s, 1H), 6.057 (d, 1H, $J=2.4 \mathrm{~Hz}), 6.076$ (s, 2H), 6.109 (s, 1H), 6.111 (d, 1H, $J=2.4 \mathrm{~Hz}), 6.126$ (s, 1H), 6.136 (s, 1H), 6.778 (d, 1H, $J=8.1), 6.796$ (d, 1H, $J=8.2), 6.815$ (d, $1 \mathrm{H}, J=8.3), 6.827$ (d, $1 \mathrm{H}, J$ $=8.3), 6.837(\mathrm{~d}, 1 \mathrm{H}, J=8.2), 6.878(\mathrm{~d}, 1 \mathrm{H}, J=8.1), 6.924(\mathrm{dd}, 1 \mathrm{H}, J=8.2,2.1), 6.935(\mathrm{dd}, 1 \mathrm{H}, J$ $=8.2,2.1), 6.962(\mathrm{dd}, 1 \mathrm{H}, J=8.2,2.1), 6.997(\mathrm{~d}, 1 \mathrm{H}, J=2.1), 7.040(\mathrm{dd}, 1 \mathrm{H}, J=8.1,2.3), 7.055$ (dd, $1 \mathrm{H}, J=8.1,2.3), 7.060$ (dd, $1 \mathrm{H}, J=8.2,2.1), 7.157$ (d, $1 \mathrm{H}, J=2.1), 7.163$ (d, 1H, $J=2.2$ ), 7.139 (d, $1 \mathrm{H}, J=2.2), 7.179(\mathrm{~d}, 1 \mathrm{H}, J=2.1), 7.204(\mathrm{~d}, 1 \mathrm{H}, J=2.1) .{ }^{13} \mathrm{C}\left\{{ }^{1} \mathrm{H}\right\}$ NMR (Methanol- $d_{4}$, $100 \mathrm{MHz}, 278 \mathrm{~K}): \delta 29.0,29.4,29.6,29.8,38.4,38.6,67.6,68.0,68.2,68.2,72.3,72.6,78.7,79.9$, 85.1, 96.1, 96.6, 96.6, 96.6, 97.4, 97.5, 98.2, 100.0, 100.1, 100.2, 103.1, 104.0, 104.2, 104.4, 105.6, 106.0, 106.2, 106.4, 106.8, 110.5, 111.0, 115.5, 115.5, 115.5, 115.5, 115.5, 115.5, 115.9, 115.9, $115.9,116.2,116.8,117.1,119.7,119.7,119.8,120.7,121.1,121.8,130.0,130.8,131.4,132.0$, $132.3,132.5,145.5,145.5,145.6,145.6,145.8,146.2,146.2,146.5,146.6,146.7,146.7,146.9$, $151.2,151.9,152.1,152.1,152.1,152.2,152.3,152.6,154.1,154.1,154.6,156.2,156.4,156.7$, 156.7, 156.8, 157.1, 158.0; HRMS (ESI) $m / z[\mathrm{M}+\mathrm{H}]^{+}$Calcd for $\mathrm{C}_{90} \mathrm{H}_{69} \mathrm{O}_{36}, 1725.3563$; Found 1725.3589 . 


\section{References}

1. Phansalkar, R. S.; Nam, J. W.; Chen, S. N.; McAlpine, J. B.; Leme, A. A.; Aydin, B.; BedranRusso, A. K.; Pauli, G. F. Centrifugal partition chromatography enables selective enrichment of trimeric and tetrameric proanthocyanidins for biomaterial development. J. Chromatogr. A 2018, 1535, 55-62.

2. Zhou, B. Y Alania, M Reis, R. Phansalkar, JW Nam, J McAlpine, SN Chen, A Bedran-Russo, G Pauli. Tri- and Tetrameric Proanthocyanidins with Dentin Bioactivities from Pinus massoniana. J. Org. Chem. 2020, in press, DOI:10.1021/acs.joc.0c00783

3. Zhou, B. Y Alania, M Reis, J McAlpine, A Bedran-Russo, G Pauli, SN Chen. Rare A-Type, New Spiro-Type, and Highly Oligomeric Proanthocyanidins from Pinus massoniana. Chemrxiv, 2020, 12168168. 
Figure S3. ${ }^{1} \mathrm{H}$ NMR spectrum of $\mathbf{1}$ in $\mathrm{CD}_{3} \mathrm{OD}(800 \mathrm{MHz}, 255 \mathrm{~K})$

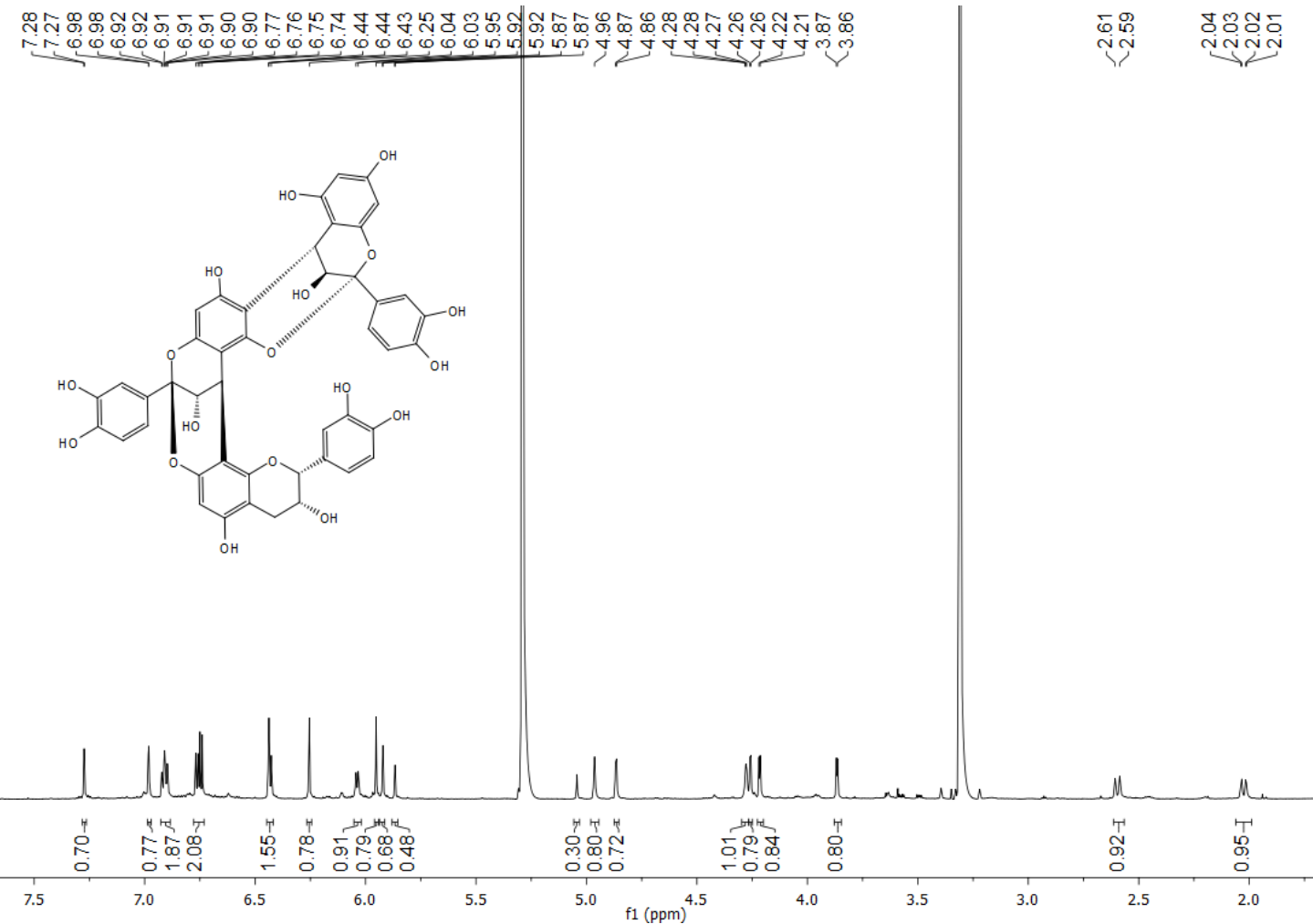

Figure S4. ${ }^{13} \mathrm{C}$ NMR spectrum of $\mathbf{1}$ in $\mathrm{CD}_{3} \mathrm{OD}(100 \mathrm{MHz}, 255 \mathrm{~K})$

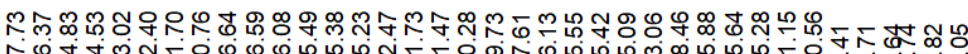

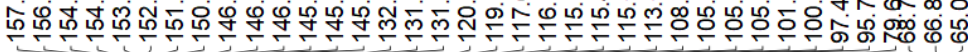
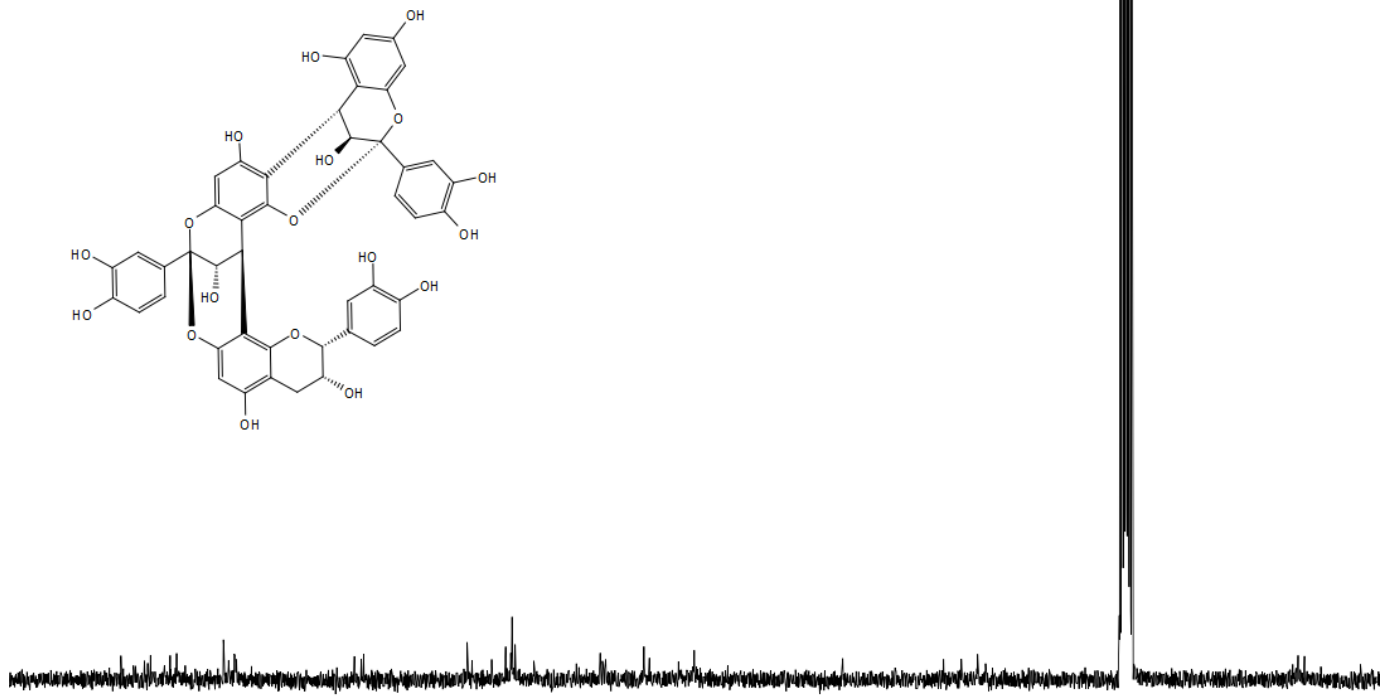

$\begin{array}{llllllllllllllllllllllllllllllll}165 & 160 & 155 & 150 & 145 & 140 & 135 & 130 & 125 & 120 & 115 & 110 & 105 & 100 & 95 & 90 & 85 & 80 & 75 & 70 & 65 & 60 & 55 & 50 & 45 & 40 & 35 & 30 & 25\end{array}$ 
Figure S5. HSQC spectrum of $\mathbf{1}$ in $\mathrm{CD}_{3} \mathrm{OD}(255 \mathrm{~K})$

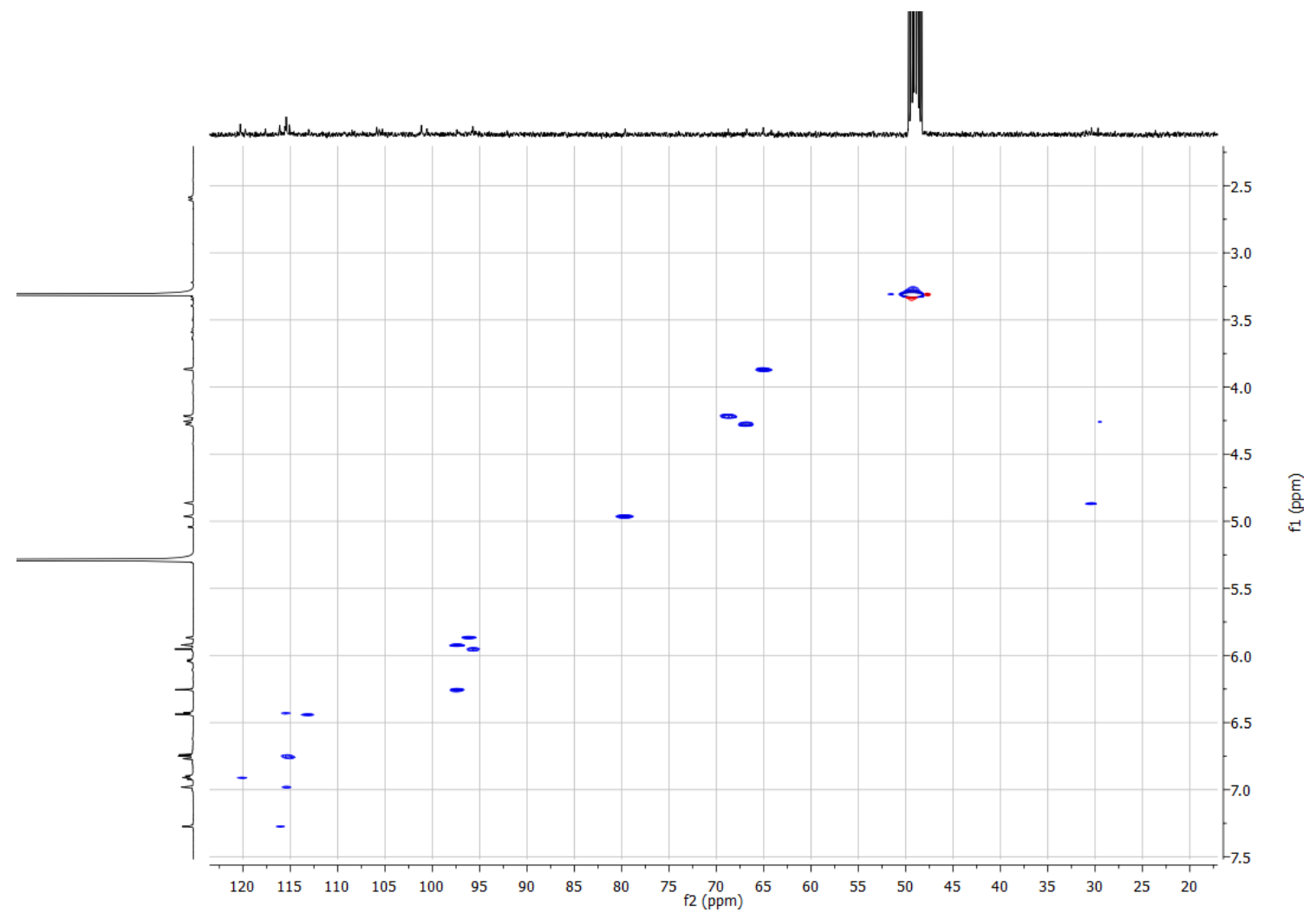

Figure S6. $\mathrm{HMBC}$ spectrum of $\mathbf{1}$ in $\mathrm{CD}_{3} \mathrm{OD}(255 \mathrm{~K})$

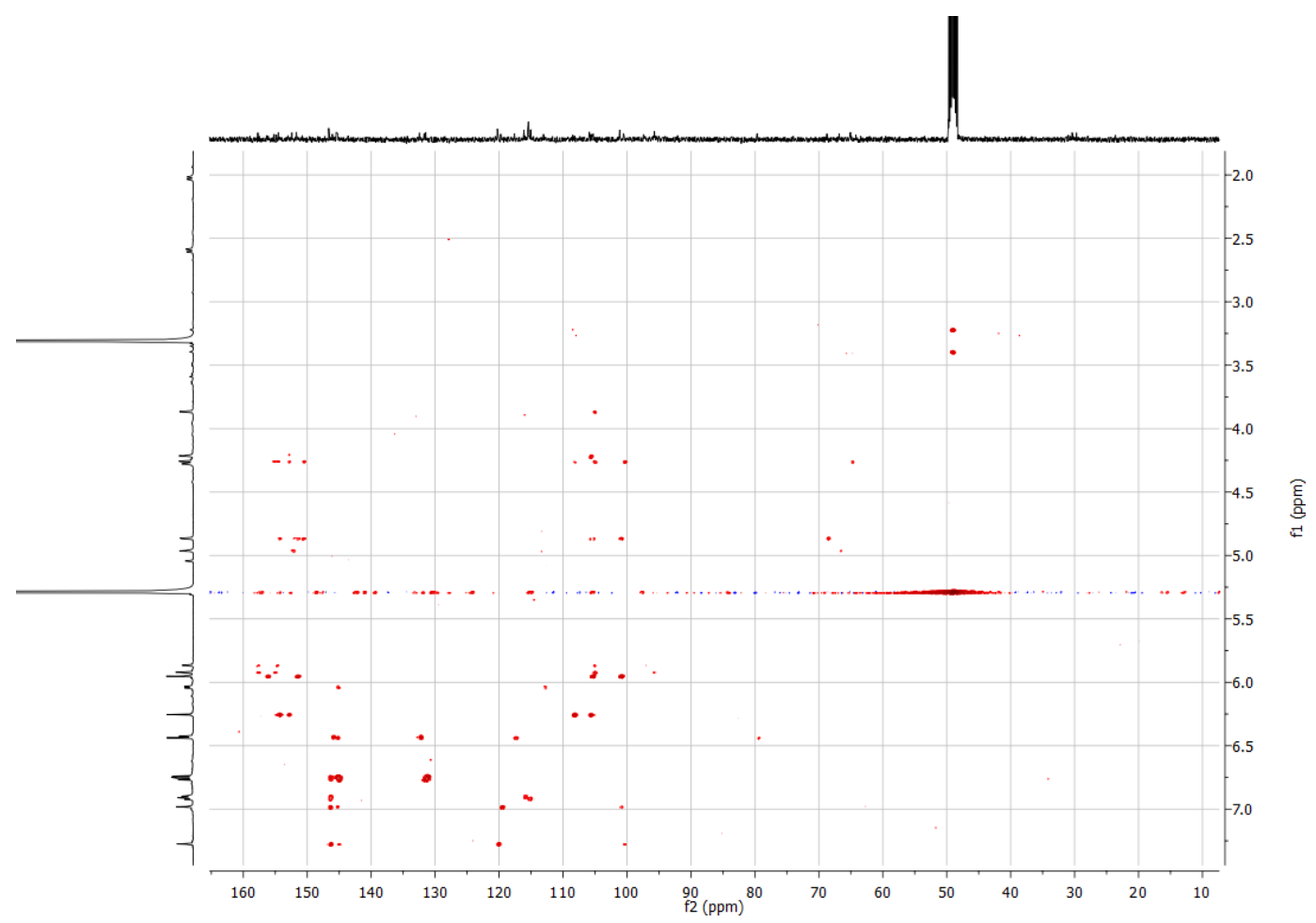


Figure S7. NOESY spectrum of $\mathbf{1}$ in $\mathrm{CD}_{3} \mathrm{OD}(255 \mathrm{~K})$

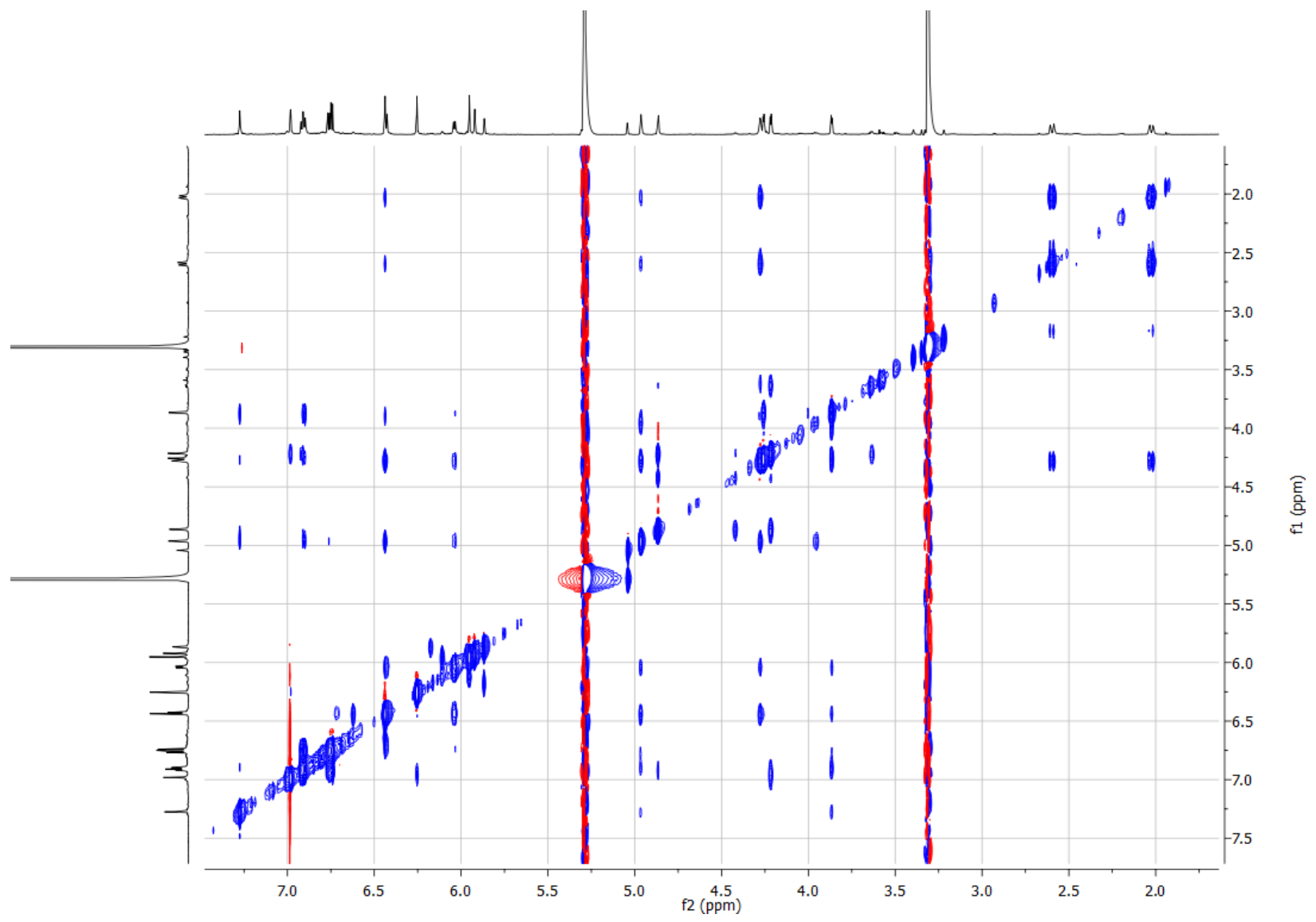

Figure S7-1. Partially expanded NOESY spectra of 1 with important NOESY correlations
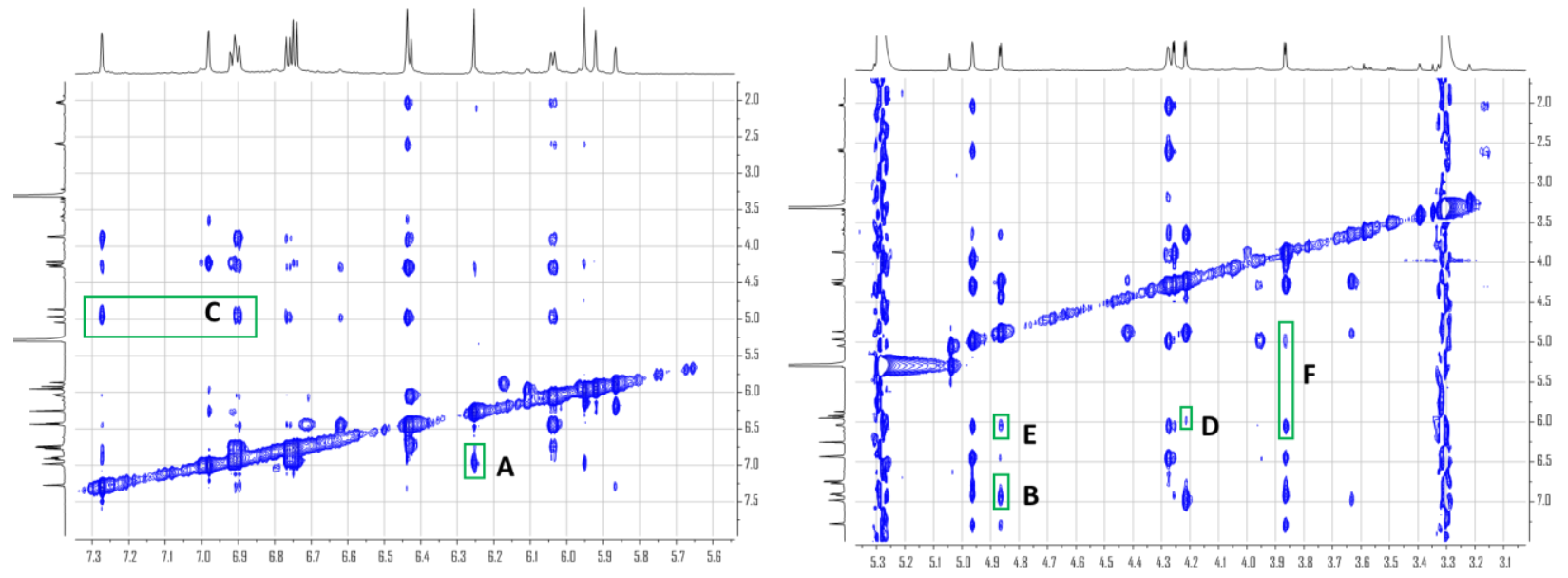
Figure S7-2. Expanded NOESY spectra of 1 with important NOESY correlations

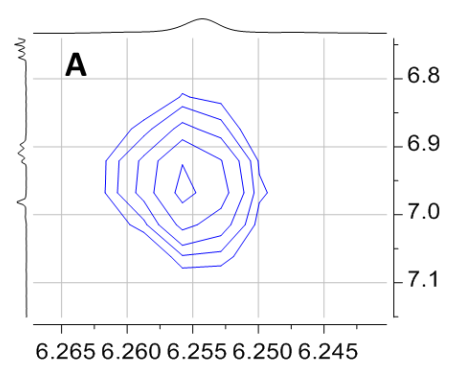

II-H-8/II-H-2' \&II-H-6'

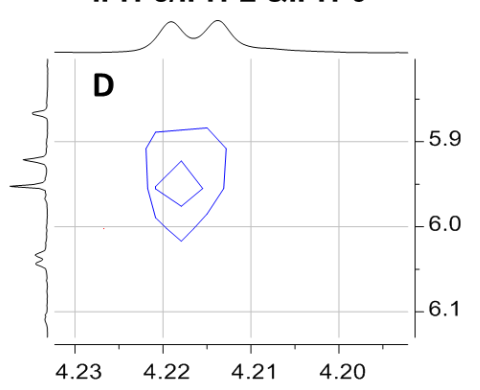

II-H-3/III-H-6

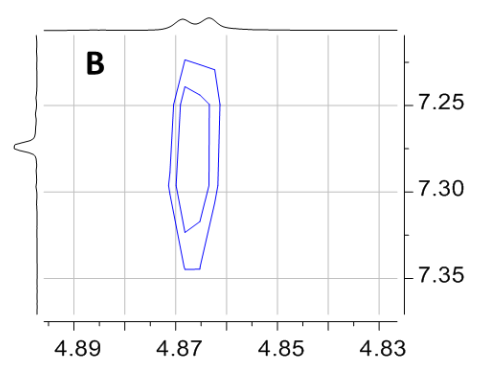

II-H-4/I-H-2'

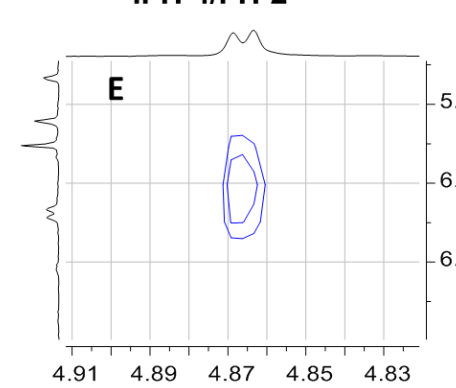

II-H-4/III-H-6'

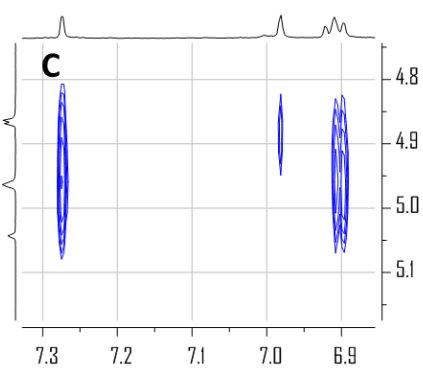

III-H-2/I-H-2' \& II-H-6'

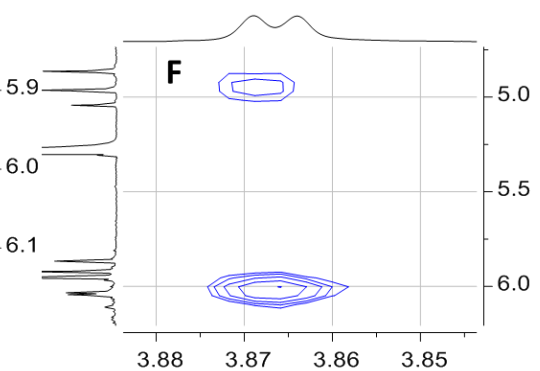

I-H-3/III-H-2\&III-H-6' 
Figure S8. (+)-HRESIMS spectrum of $\mathbf{1}$

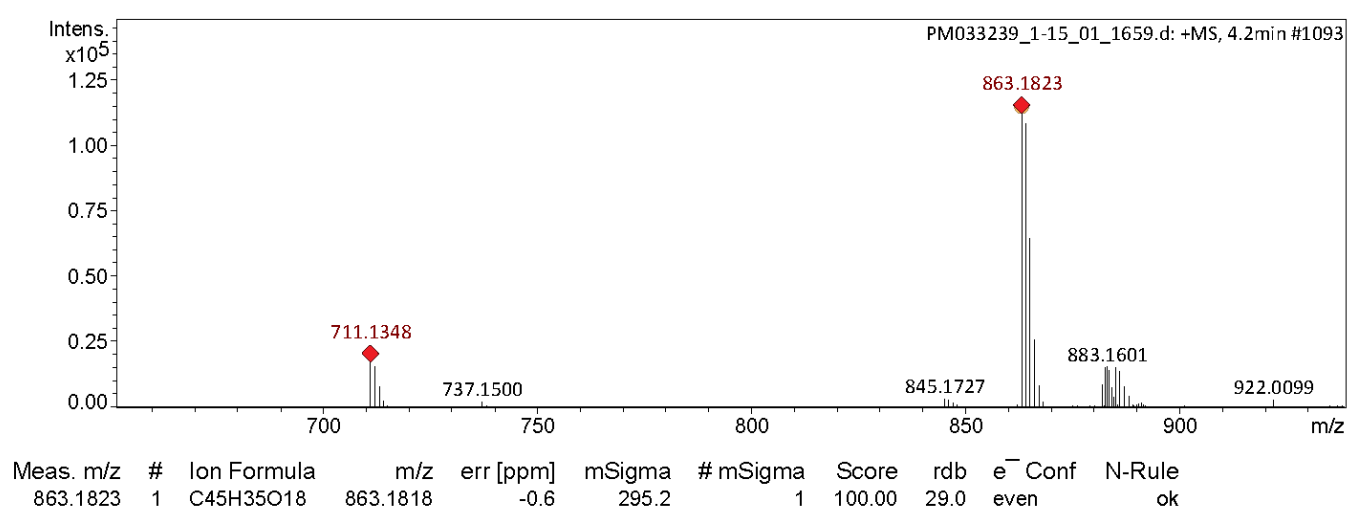

Figure S9. ${ }^{1} \mathrm{H}$ NMR spectrum of 2 in $\mathrm{CD}_{3} \mathrm{OD}(800 \mathrm{MHz}, 255 \mathrm{~K})$

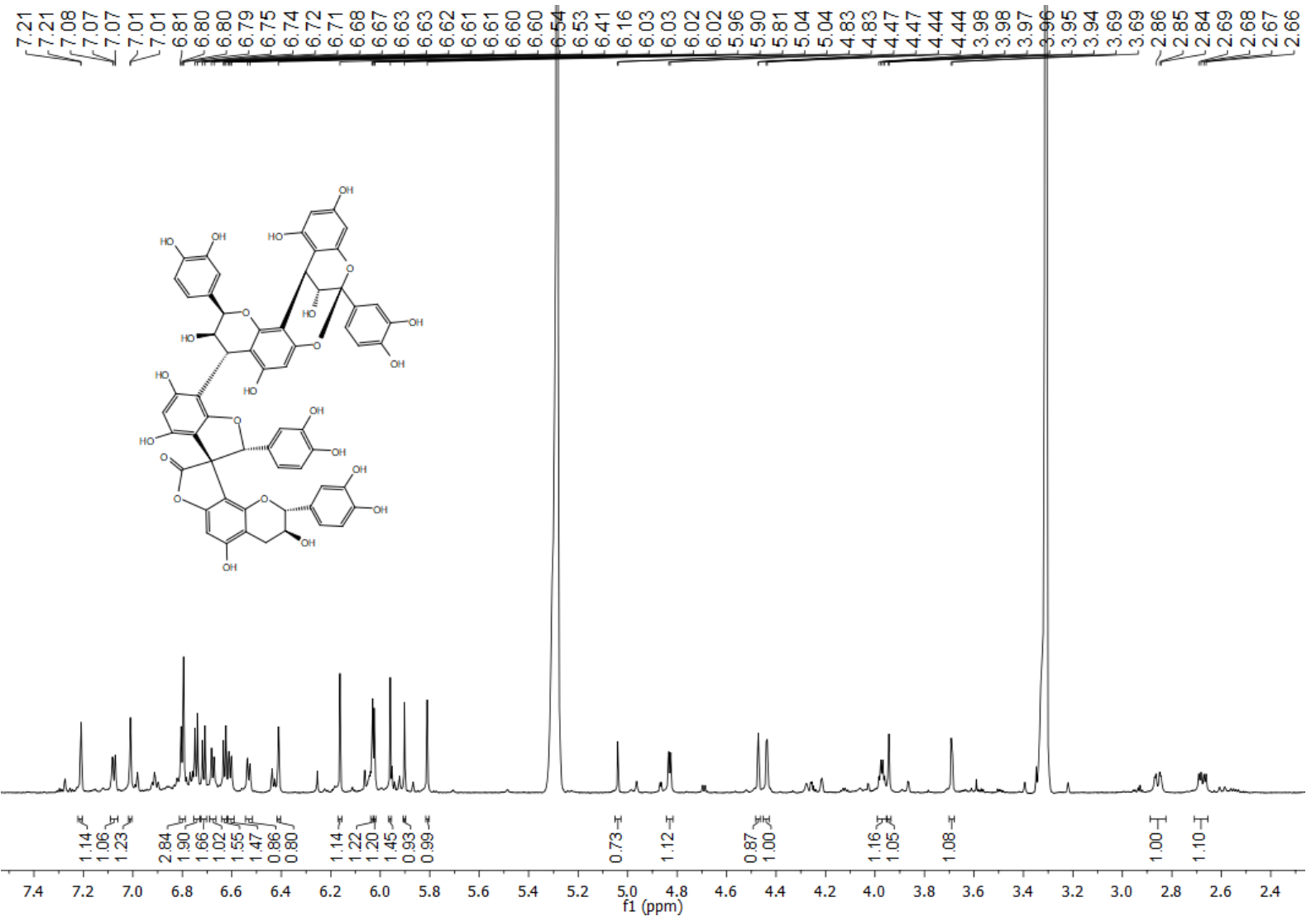


Figure S10. Comparison of the ${ }^{1} \mathrm{H}$ NMR spectra of 2 in $\mathrm{CD}_{3} \mathrm{OD}$ at $278 \mathrm{~K}$ and $255 \mathrm{~K}(800 \mathrm{MHz})$

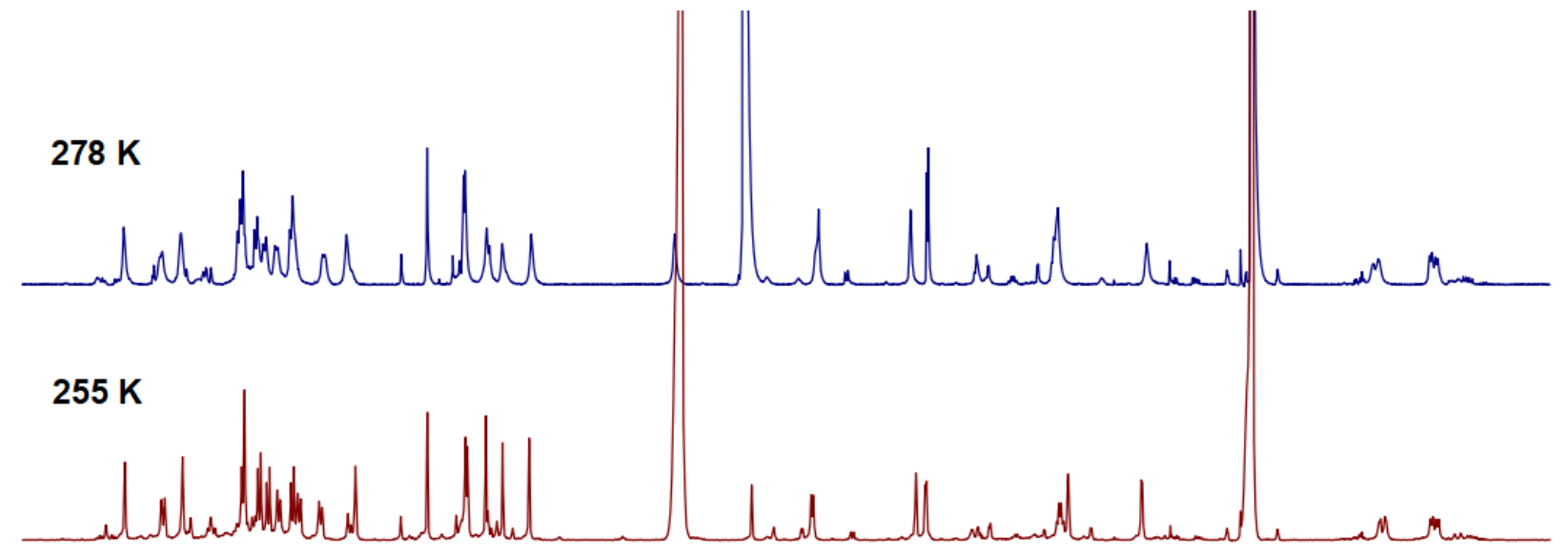

Figure S11. ${ }^{13} \mathrm{C}$ NMR spectrum of 2 in $\mathrm{CD}_{3} \mathrm{OD}(100 \mathrm{MHz}, 255 \mathrm{~K})$

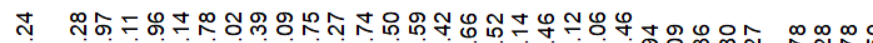

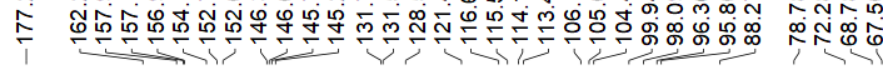

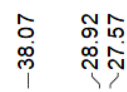
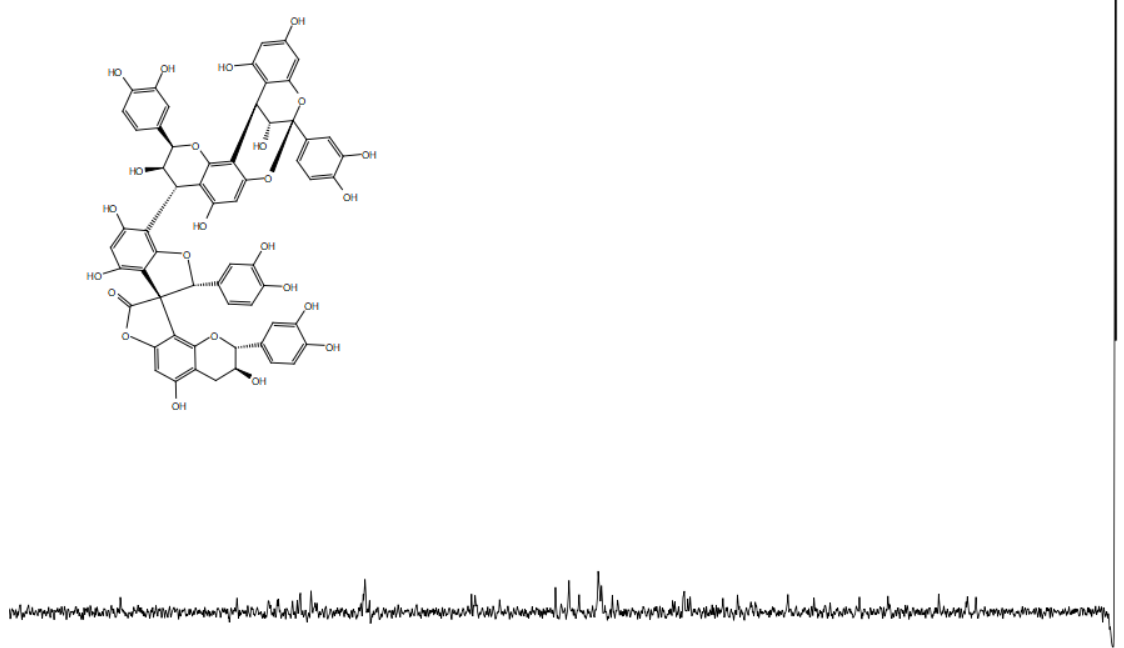

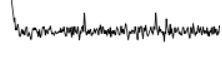

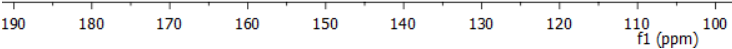


Figure S12. ${ }^{1} \mathrm{H}-{ }^{1} \mathrm{HCOSY}$ spectrum of 2 in $\mathrm{CD}_{3} \mathrm{OD}(255 \mathrm{~K})$

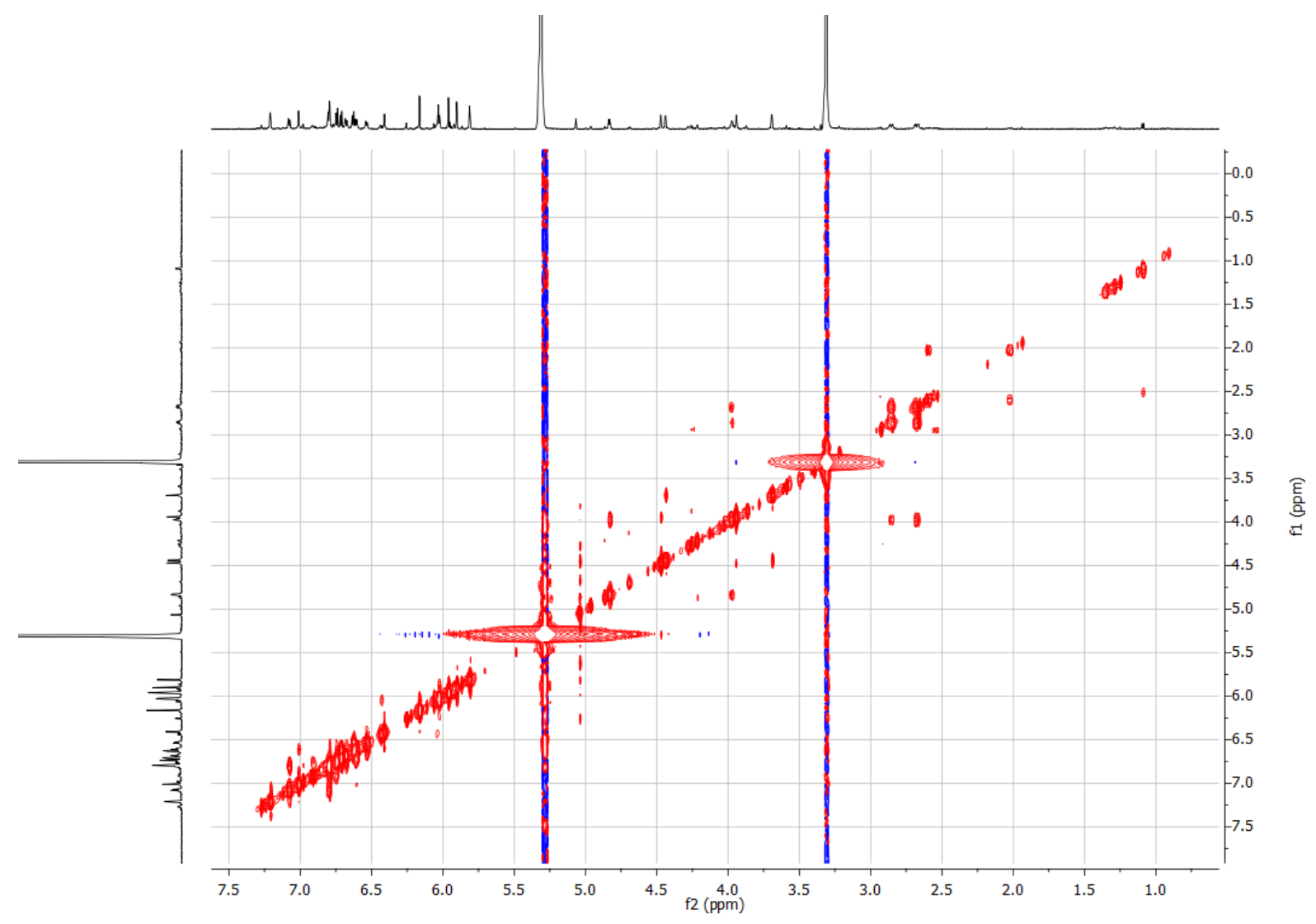

Figure S13. HSQC spectrum of 2 in $\mathrm{CD}_{3} \mathrm{OD}(255 \mathrm{~K})$

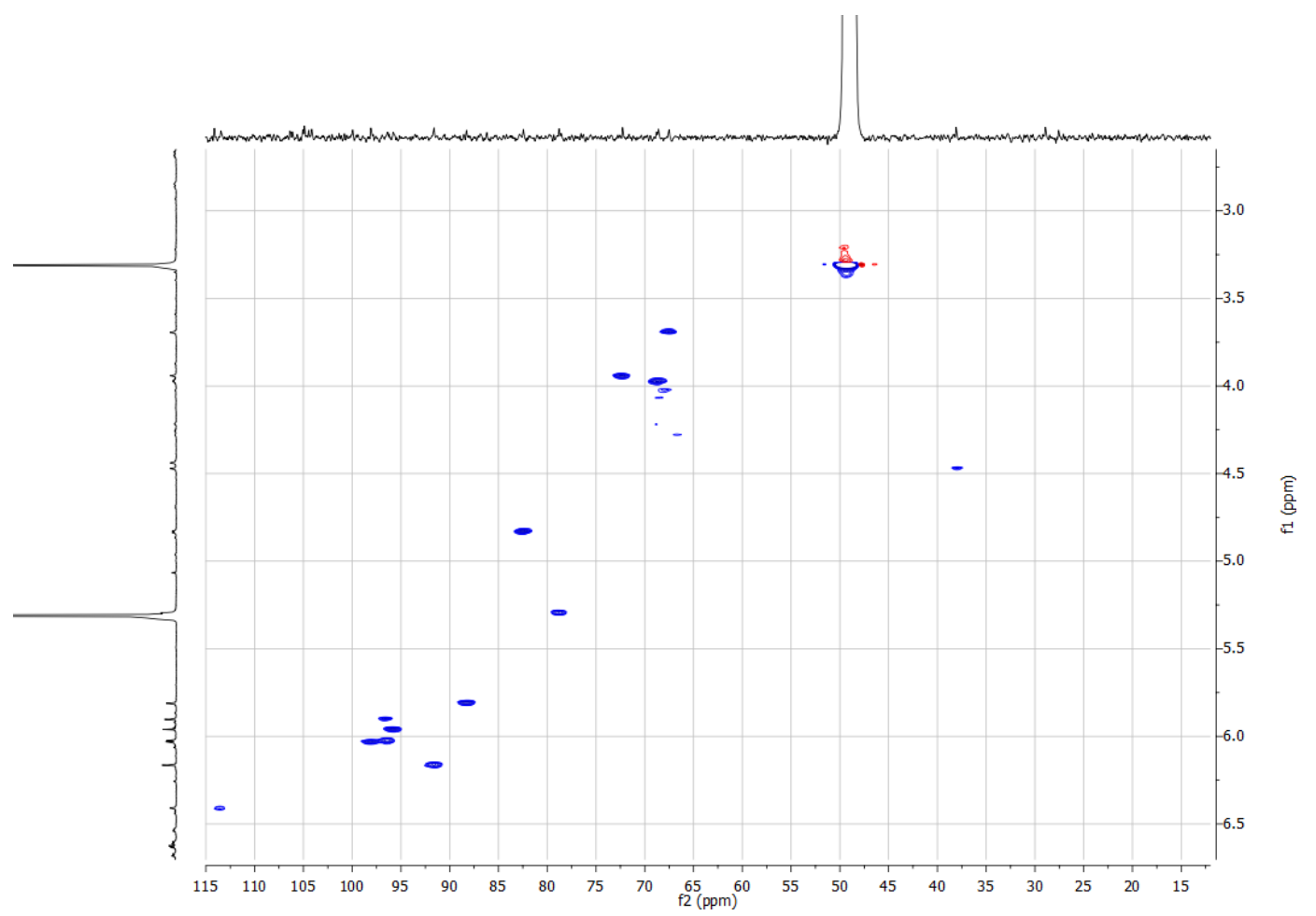

15 
Figure S14. $\mathrm{HMBC}$ spectrum of 2 in $\mathrm{CD}_{3} \mathrm{OD}(255 \mathrm{~K})$

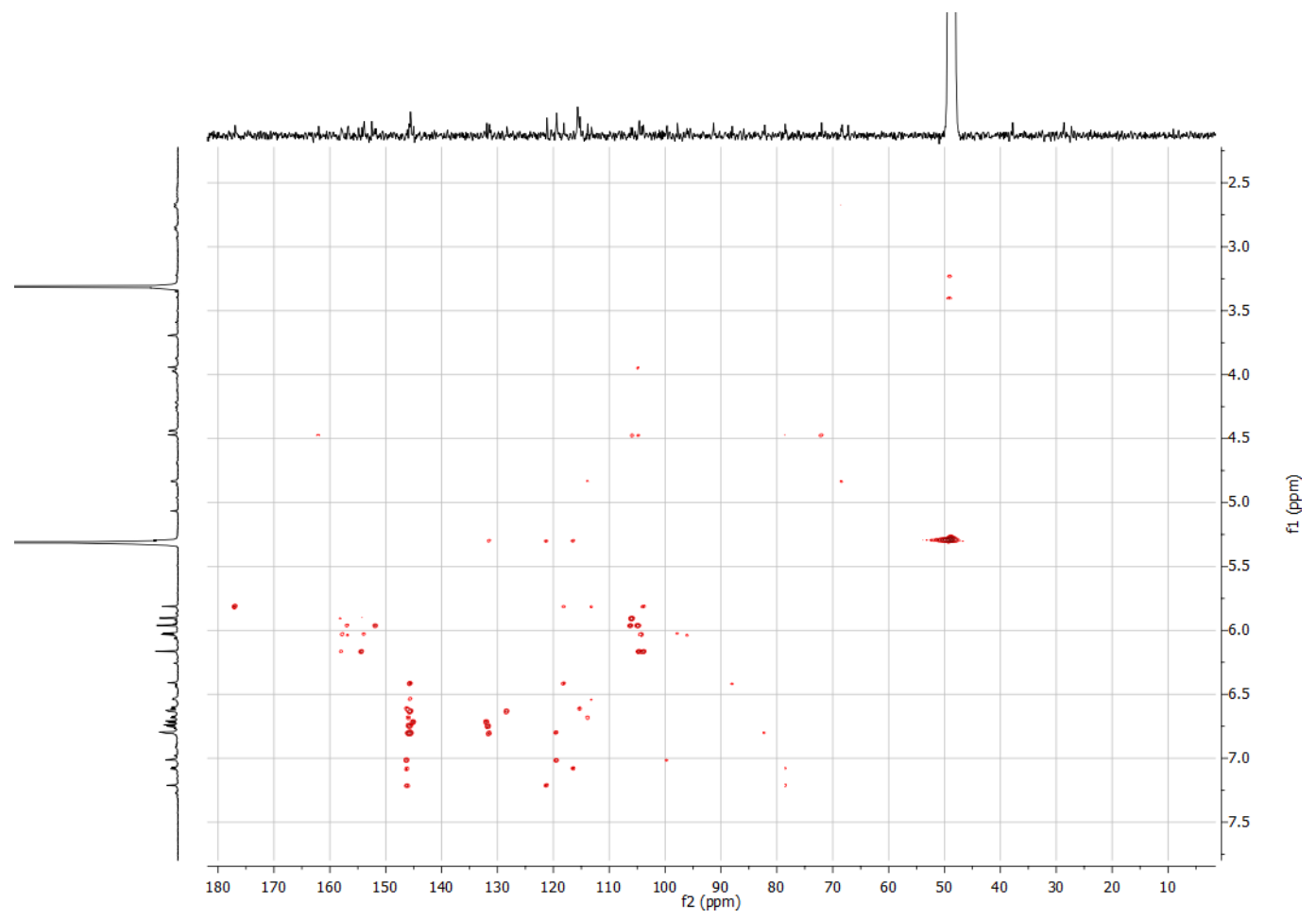

Figure S15. NOESY spectrum of 2 in $\mathrm{CD}_{3} \mathrm{OD}(255 \mathrm{~K})$

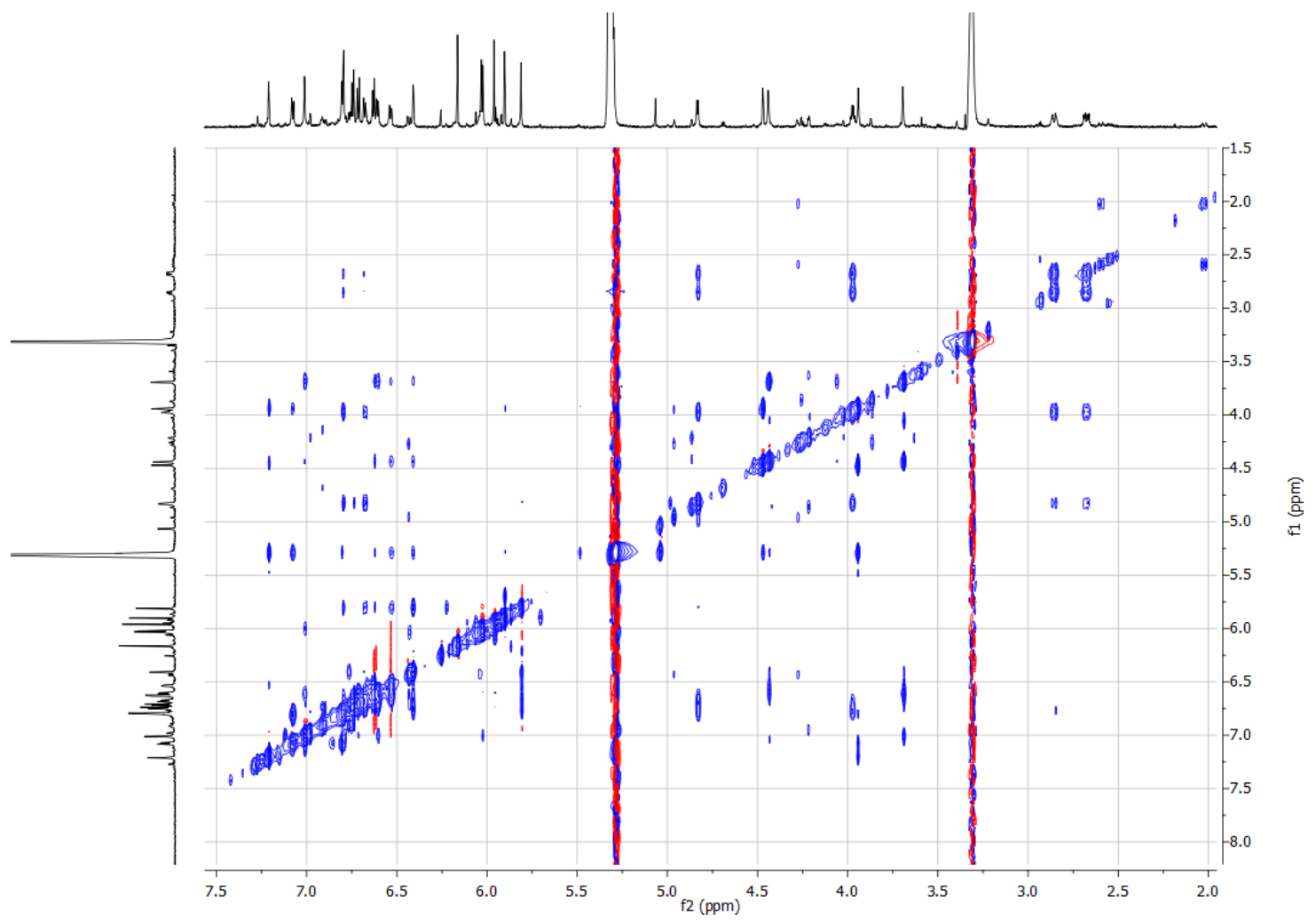


Figure S15-1. Partially expanded NOESY spectra of 2 with important NOESY correlations

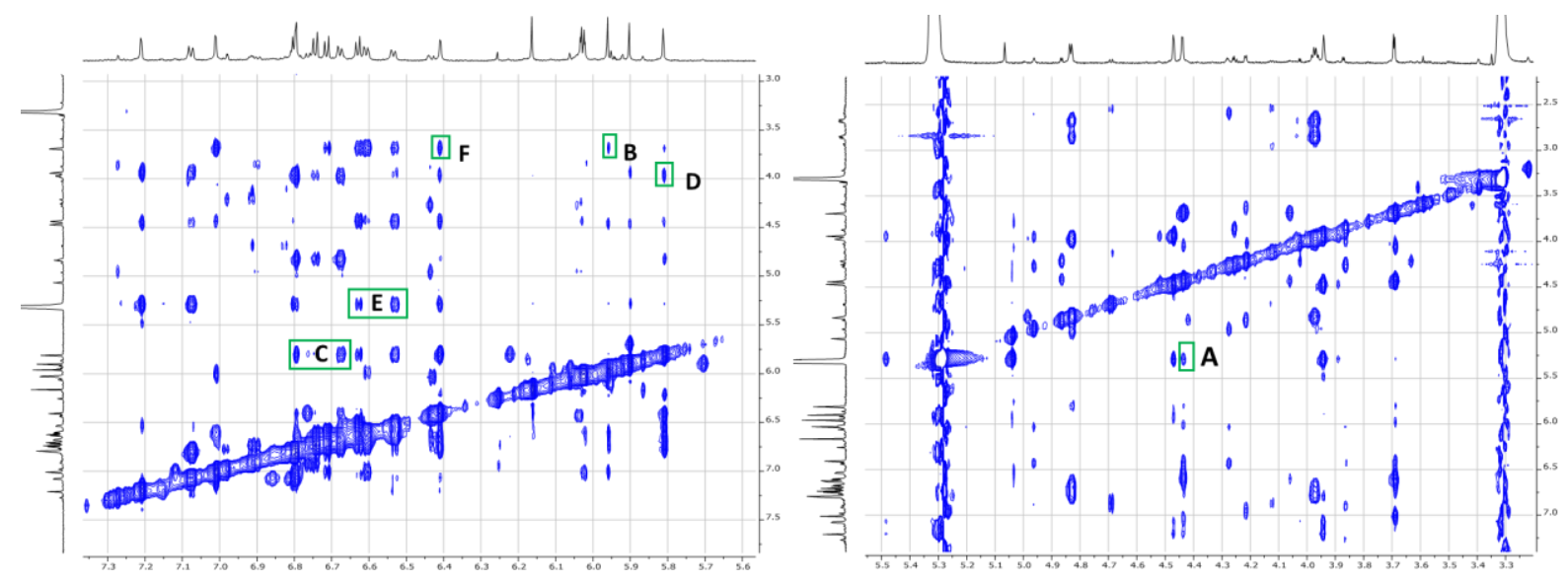

Figure S15-2. Expanded NOESY spectra of 2 with important NOESY correlations
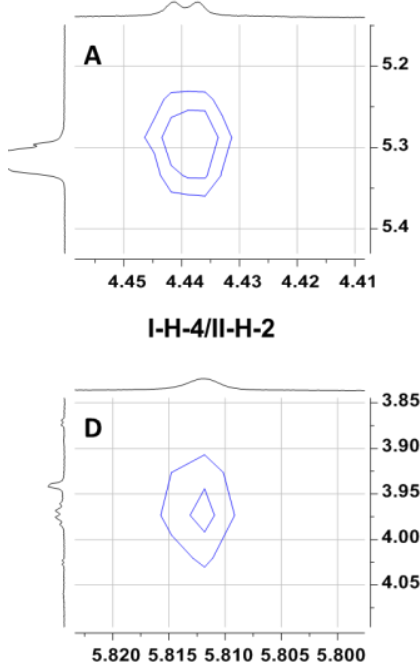

III-H-2/IV-H-3

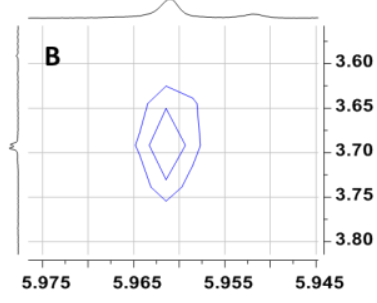

I-H-3/II-H-6

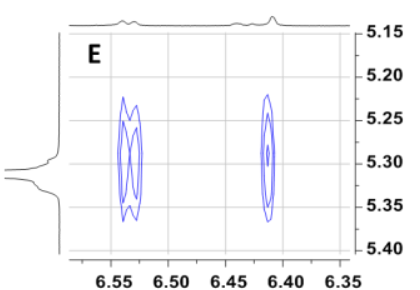

II-H-2/III-H-2'\&III-H-6'
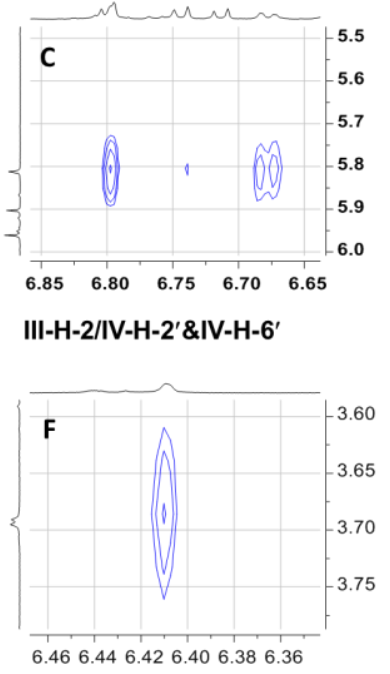

I-H-3/III-H-2

Figure S16. (+)-HRESIMS spectrum of 2

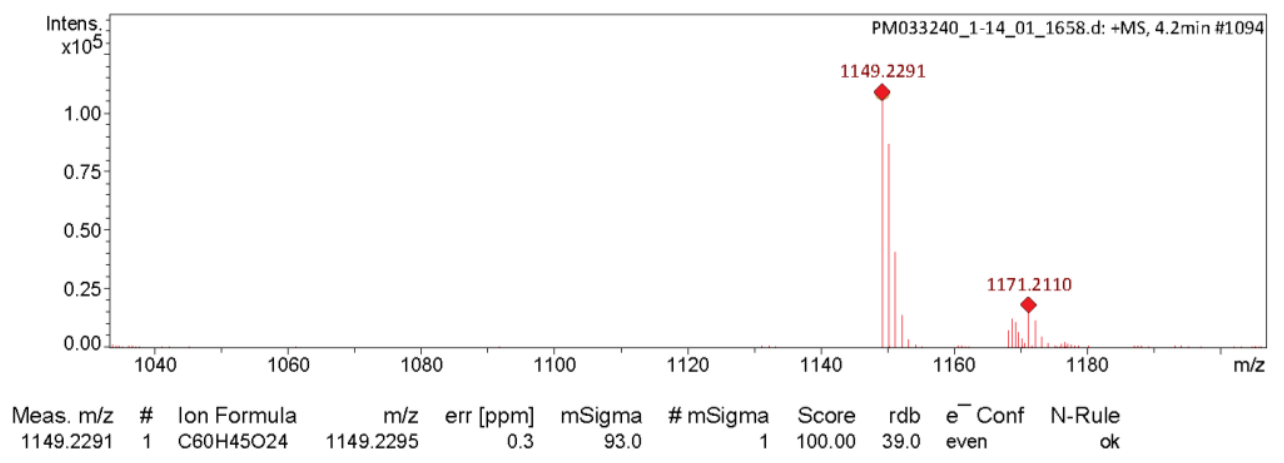


Figure S17. ${ }^{1} \mathrm{H}$ NMR spectrum of 3 in $\mathrm{CD}_{3} \mathrm{OD}(800 \mathrm{MHz}, 278 \mathrm{~K})$.

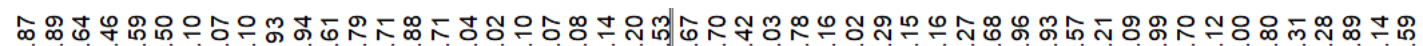

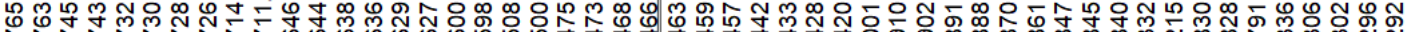

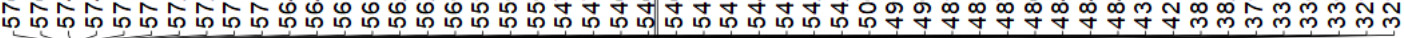

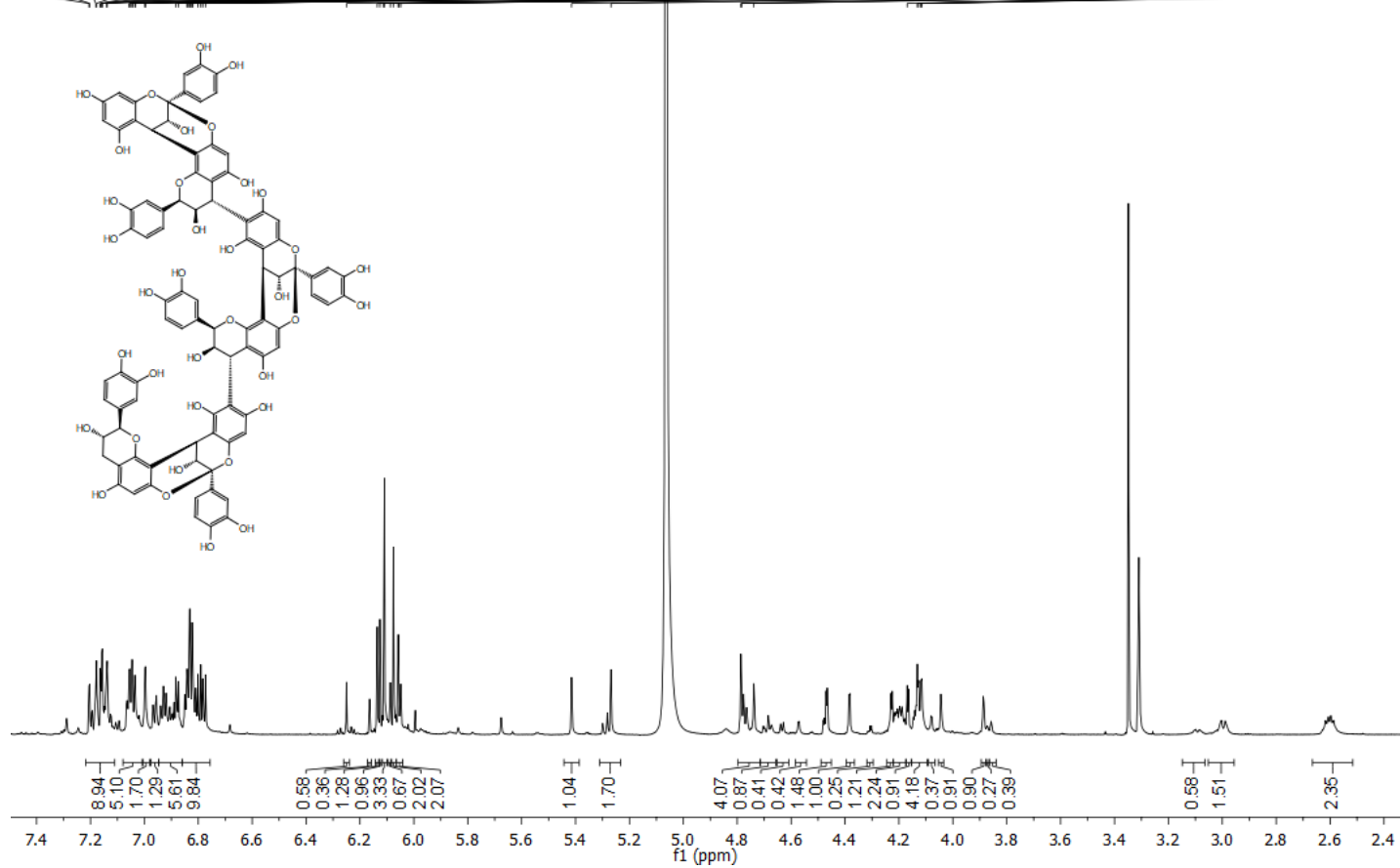

Figure S18. ${ }^{13} \mathrm{C}$ NMR spectrum of 3 in $\mathrm{CD}_{3} \mathrm{OD}(100 \mathrm{MHz}, 278 \mathrm{~K})$.

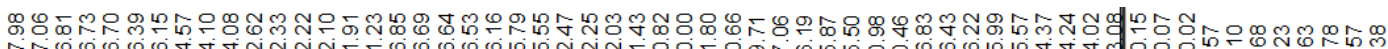

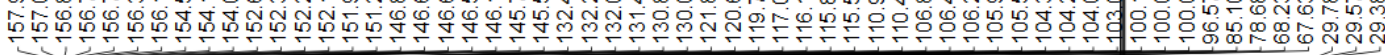
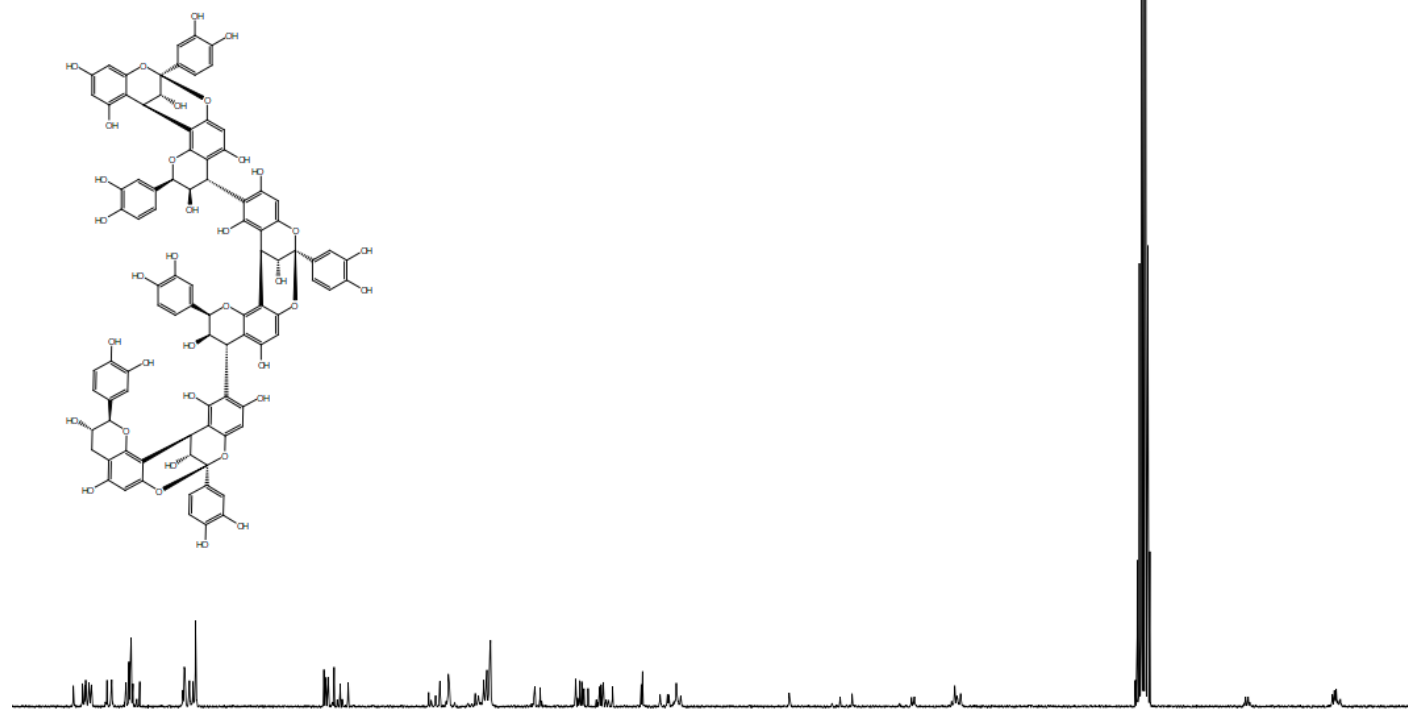

$\begin{array}{llllllllllllll}160 & 155 & 150 & 145 & 140 & 135 & 130 & 125 & 120 & 115 & 110 & 105 & 100 & 95 \\ \mathrm{f} 1(\mathrm{ppm}) & 90\end{array}$ 
Figure S19. ${ }^{1} \mathrm{H}-{ }^{1} \mathrm{HCOSY}$ spectrum of 3 in $\mathrm{CD}_{3} \mathrm{OD}(278 \mathrm{~K})$.

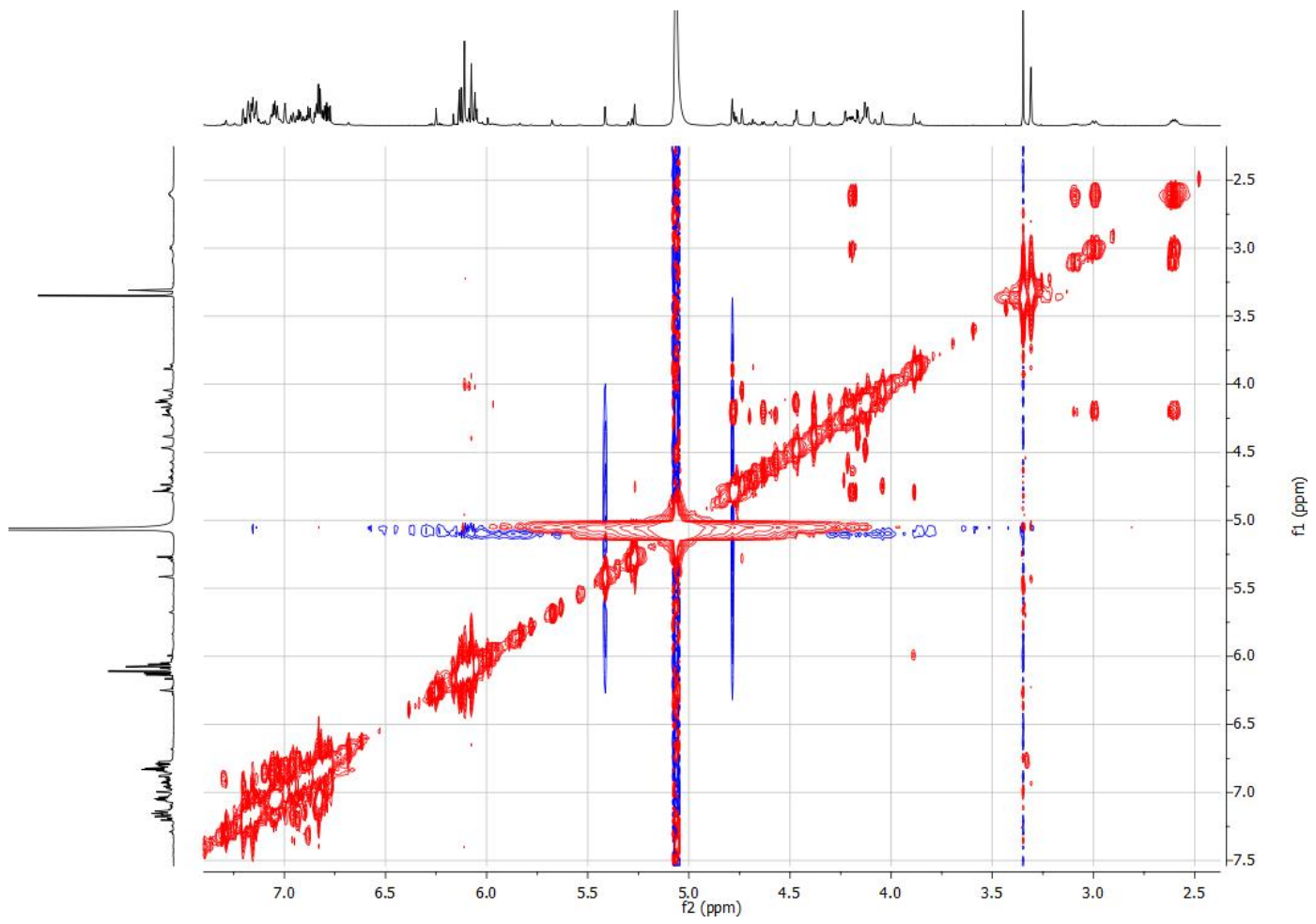

Figure S20. HSQC spectrum of $\mathbf{3}$ in $\mathrm{CD}_{3} \mathrm{OD}(278 \mathrm{~K})$.

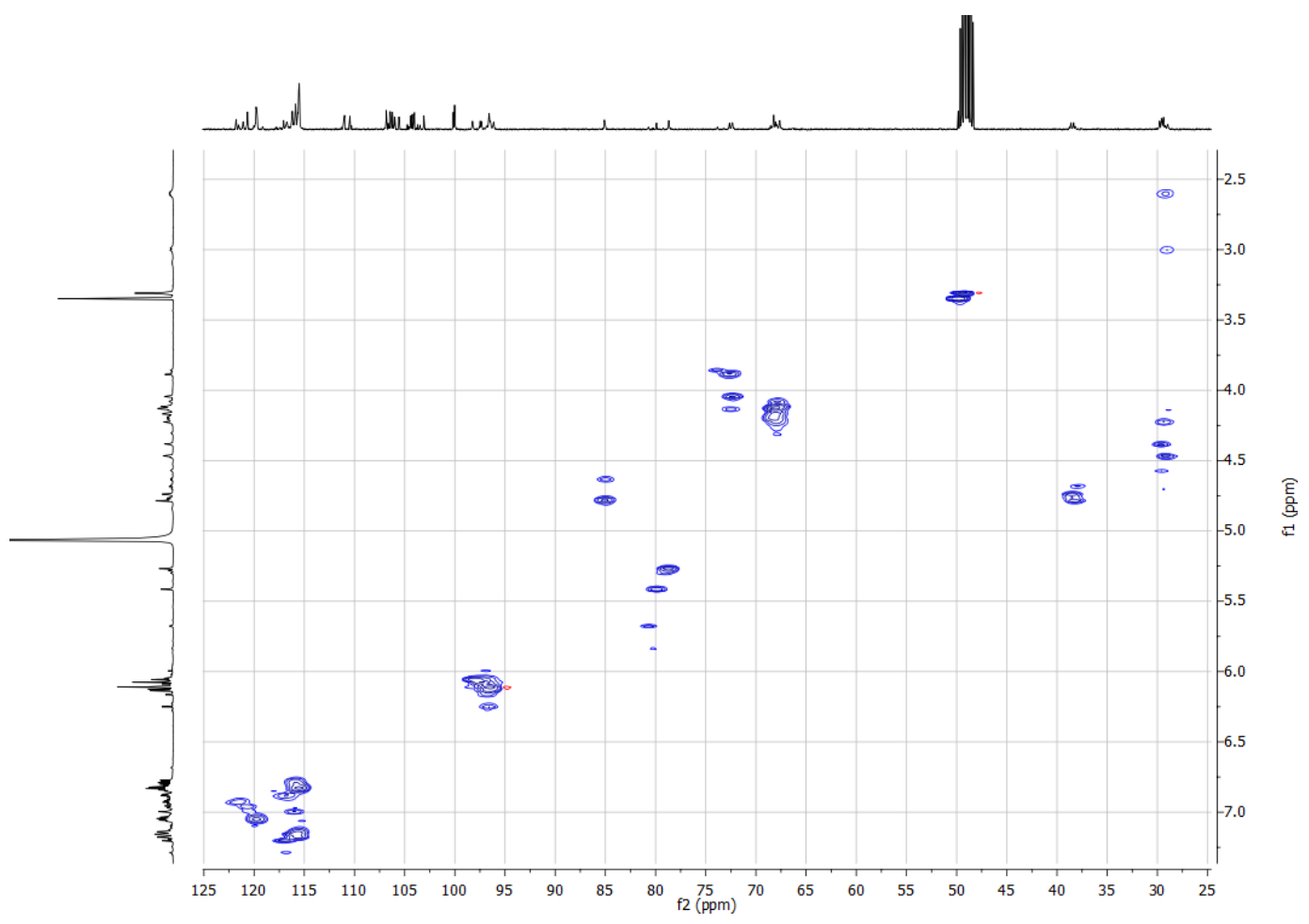


Figure S21. $\mathrm{HMBC}$ spectrum of $\mathbf{3}$ in $\mathrm{CD}_{3} \mathrm{OD}(278 \mathrm{~K})$

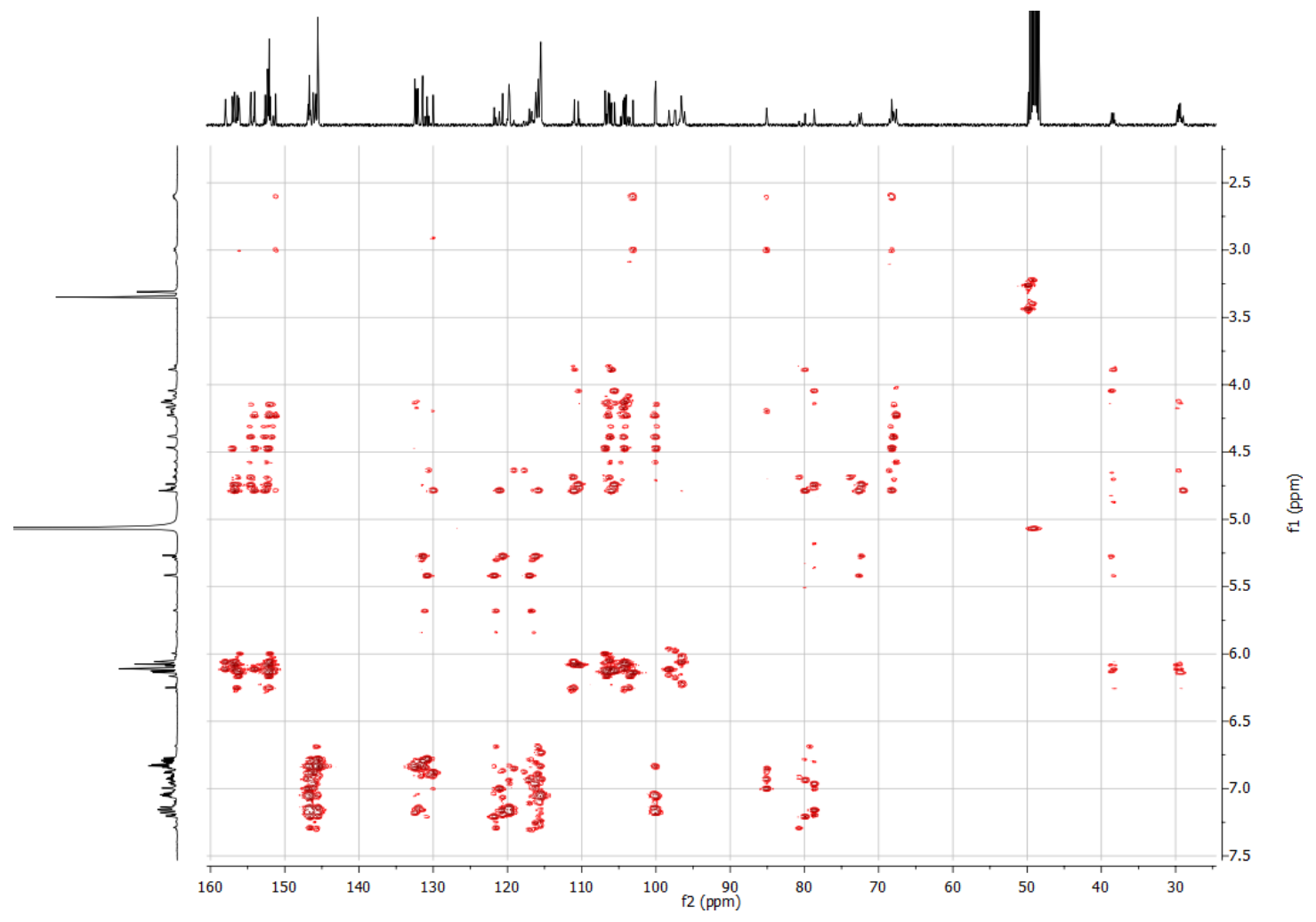

Figure S22. NOESY spectrum of $\mathbf{3}$ in $\mathrm{CD}_{3} \mathrm{OD}(278 \mathrm{~K})$

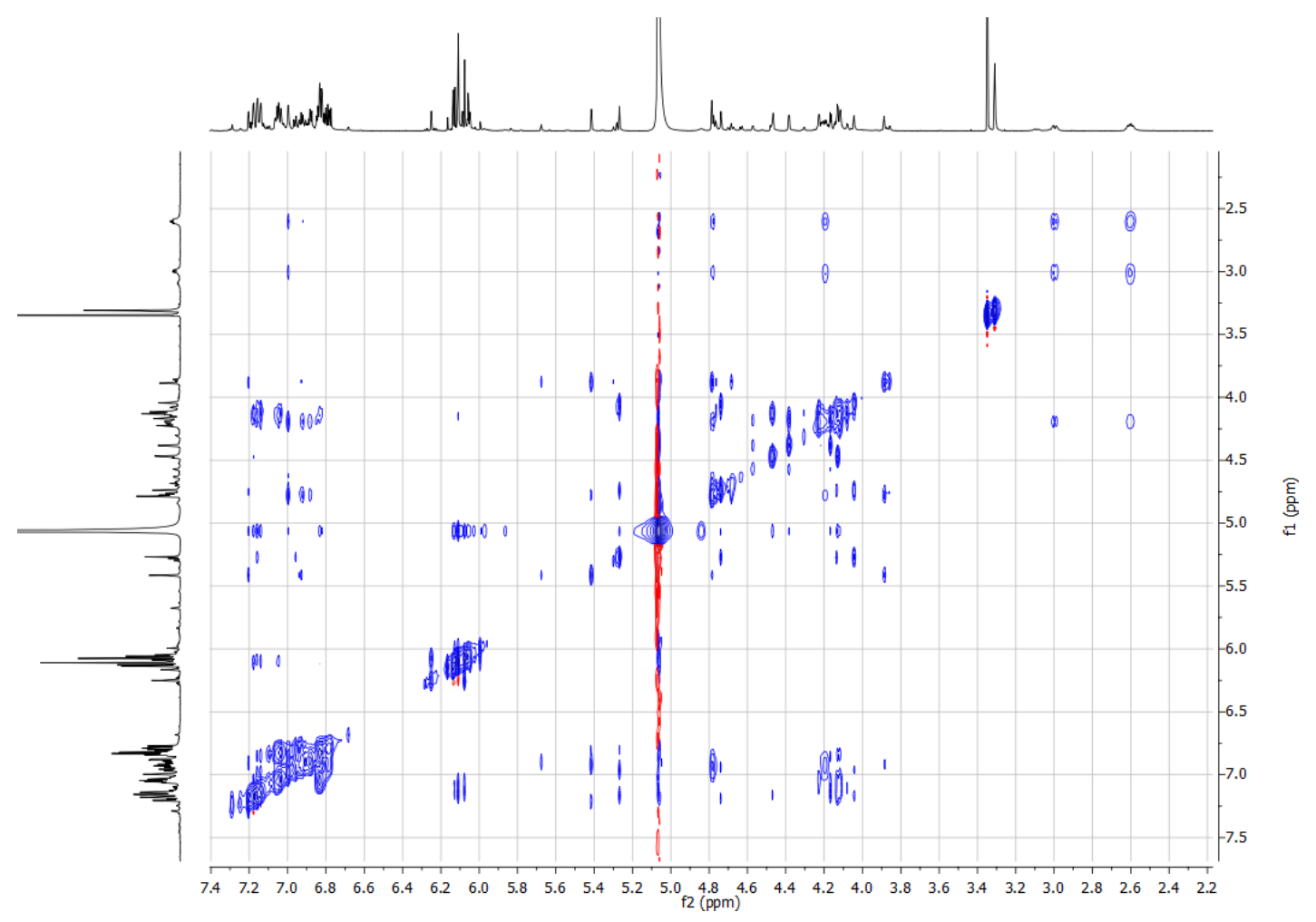


Figure S22-1. Partially expanded NOESY spectra of $\mathbf{3}$ with important NOESY correlations
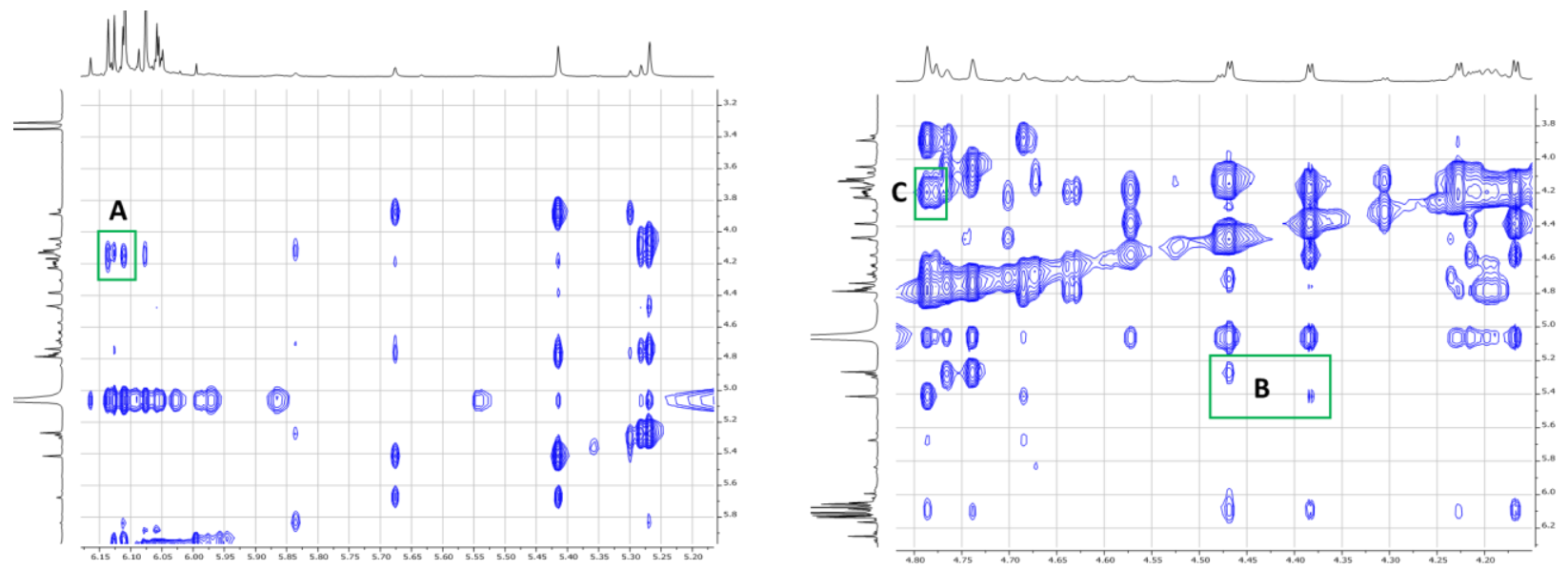

Figure S22-2. Expanded NOESY spectra of $\mathbf{3}$ with important NOESY correlations

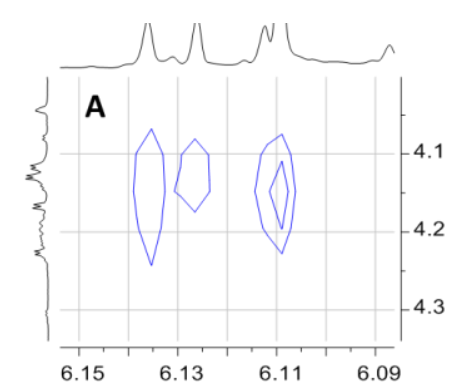

I-H-3/II-H-6

III-H-3/IV-H-6

V-H-3/VI-H-6

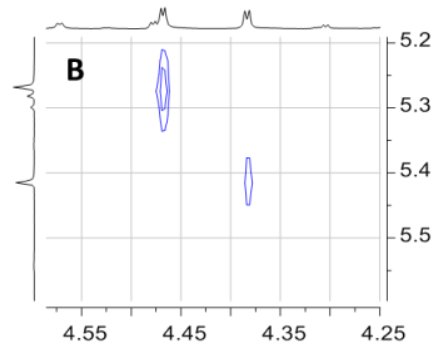

I-H-4/II-H-2

III-H-4/IV-H-2

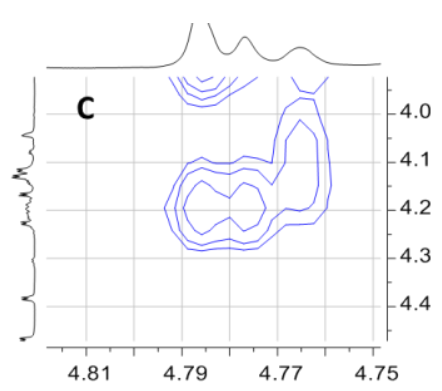

V-H-4/VI-H-2

Figure S23. (+)-HRESIMS spectrum of $\mathbf{3}$

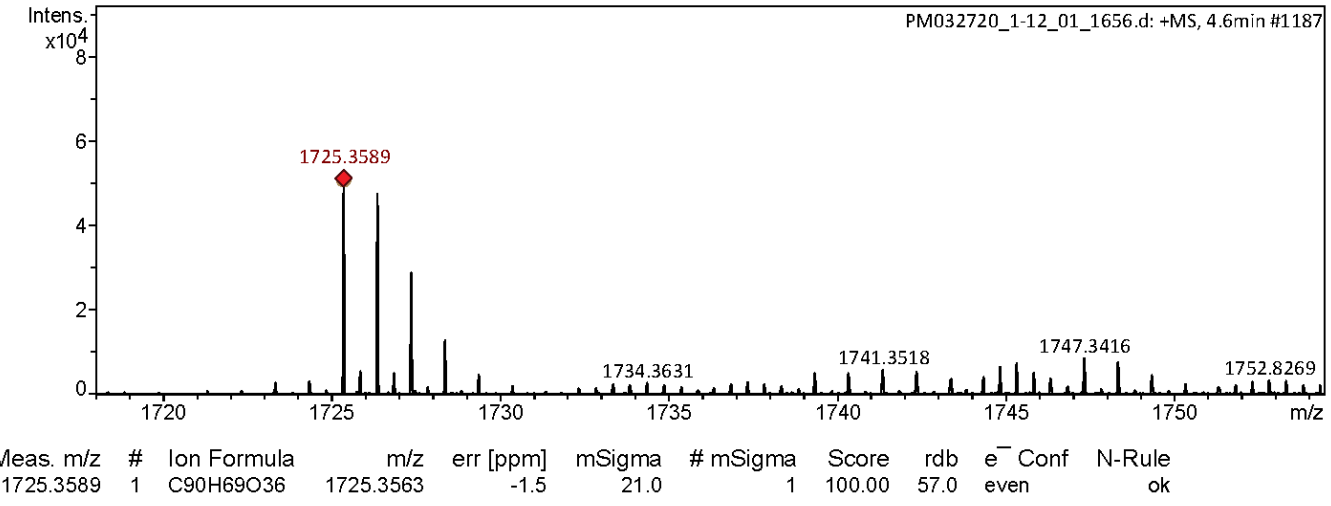


Figure S24. Phloroglucinolysis products of $\mathbf{3}$ identified by chiral phase HPLC and MS analysis

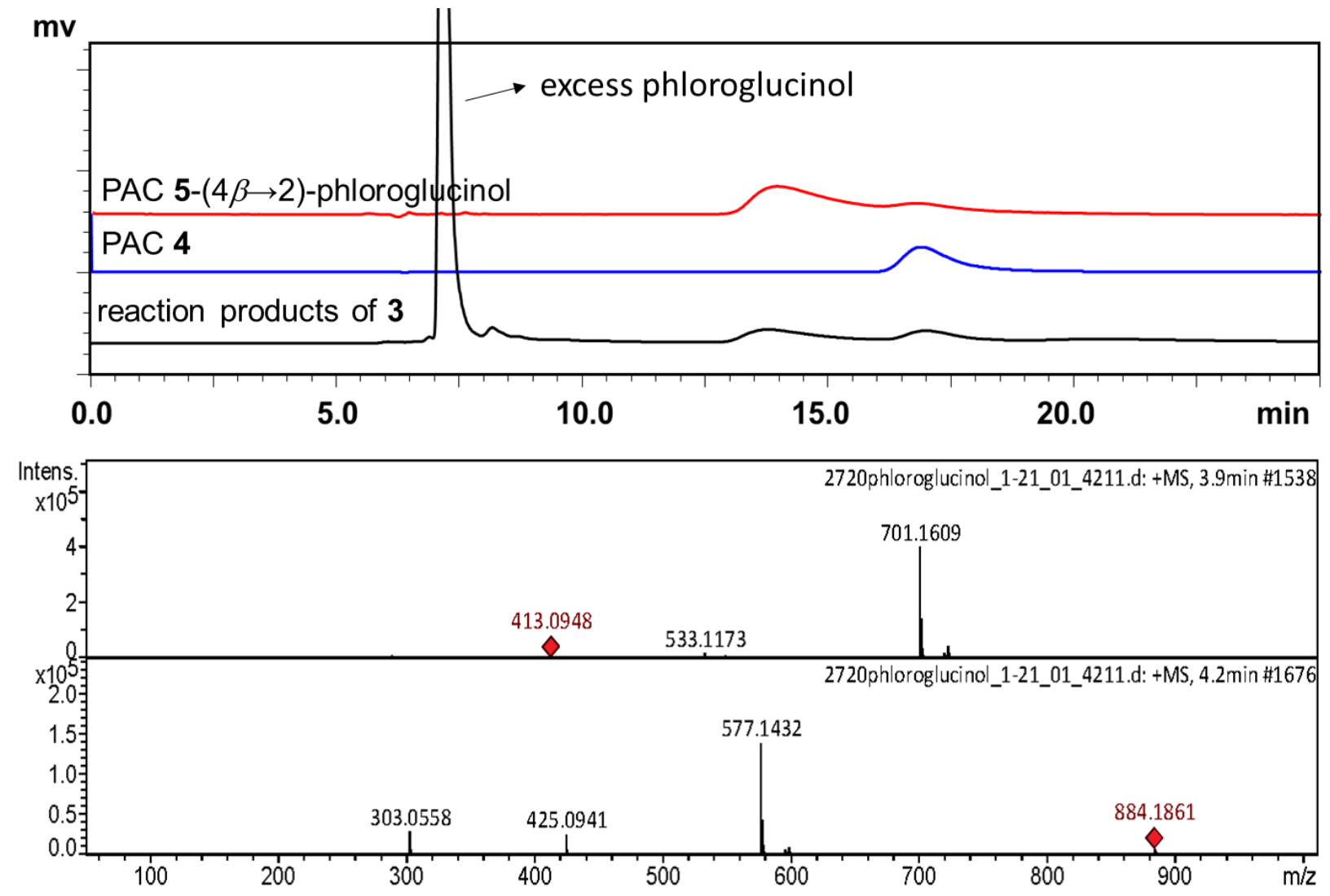


Figure S25. Calculation of the purity of $\mathbf{1}$ by qHNMR using the $100 \%$ method

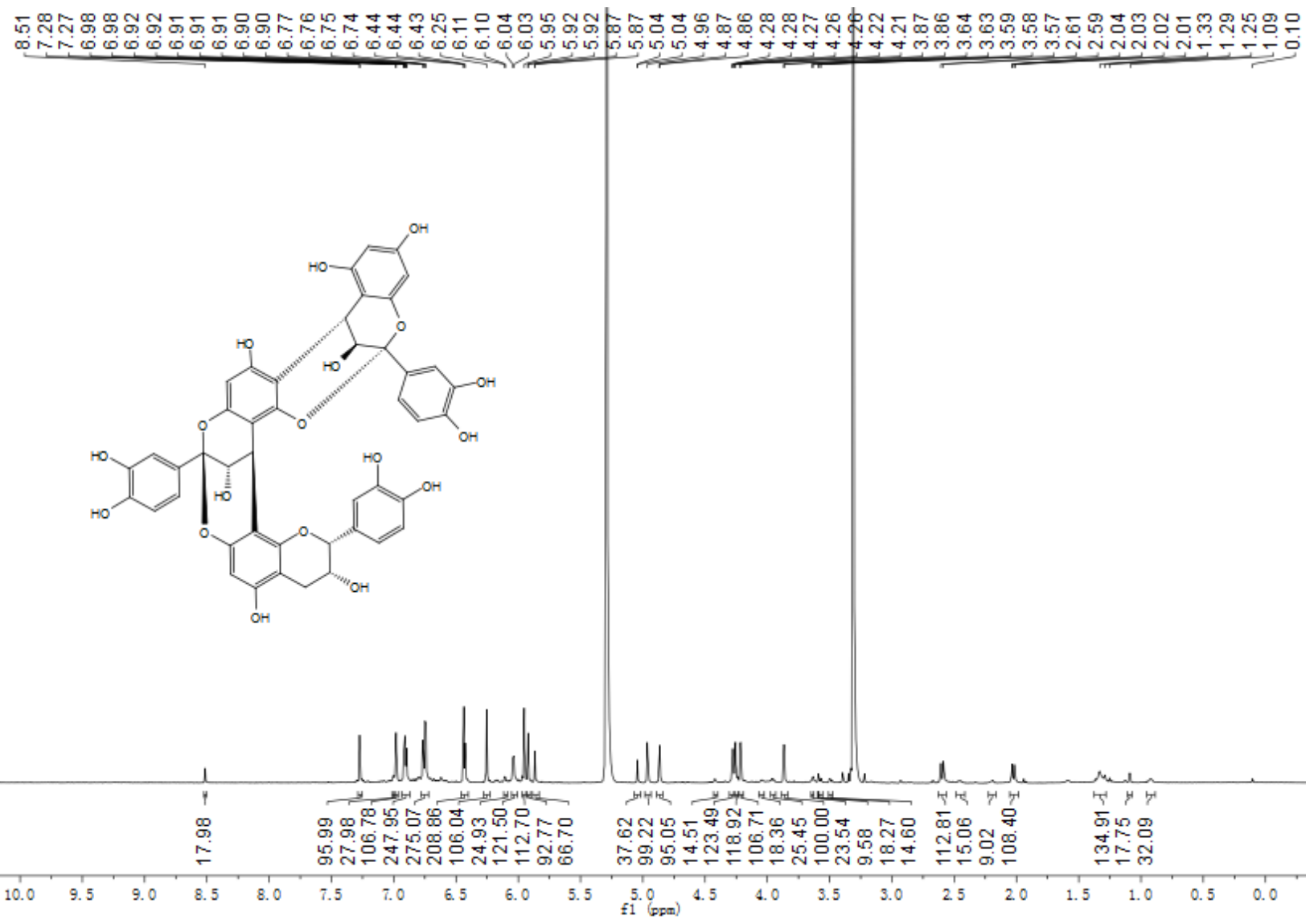

Quantitative ${ }^{1} \mathrm{H}$ NMR spectrum (800 MHz, $\mathrm{CD}_{3} \mathrm{OD}, 255 \mathrm{~K}$ )

\begin{tabular}{|l|r|}
\hline \multicolumn{2}{|c|}{ Compound 1 } \\
\hline Composition & Weight $\%$ \\
\hline Compound 1 & 88.7 \\
\hline Rotamers and/or analogues & 8.9 \\
\hline Formic acid & 0.9 \\
\hline Other impurities & 1.5 \\
\hline
\end{tabular}


Figure S26. Calculation of the purity of $\mathbf{2}$ by qHNMR using the $100 \%$ method

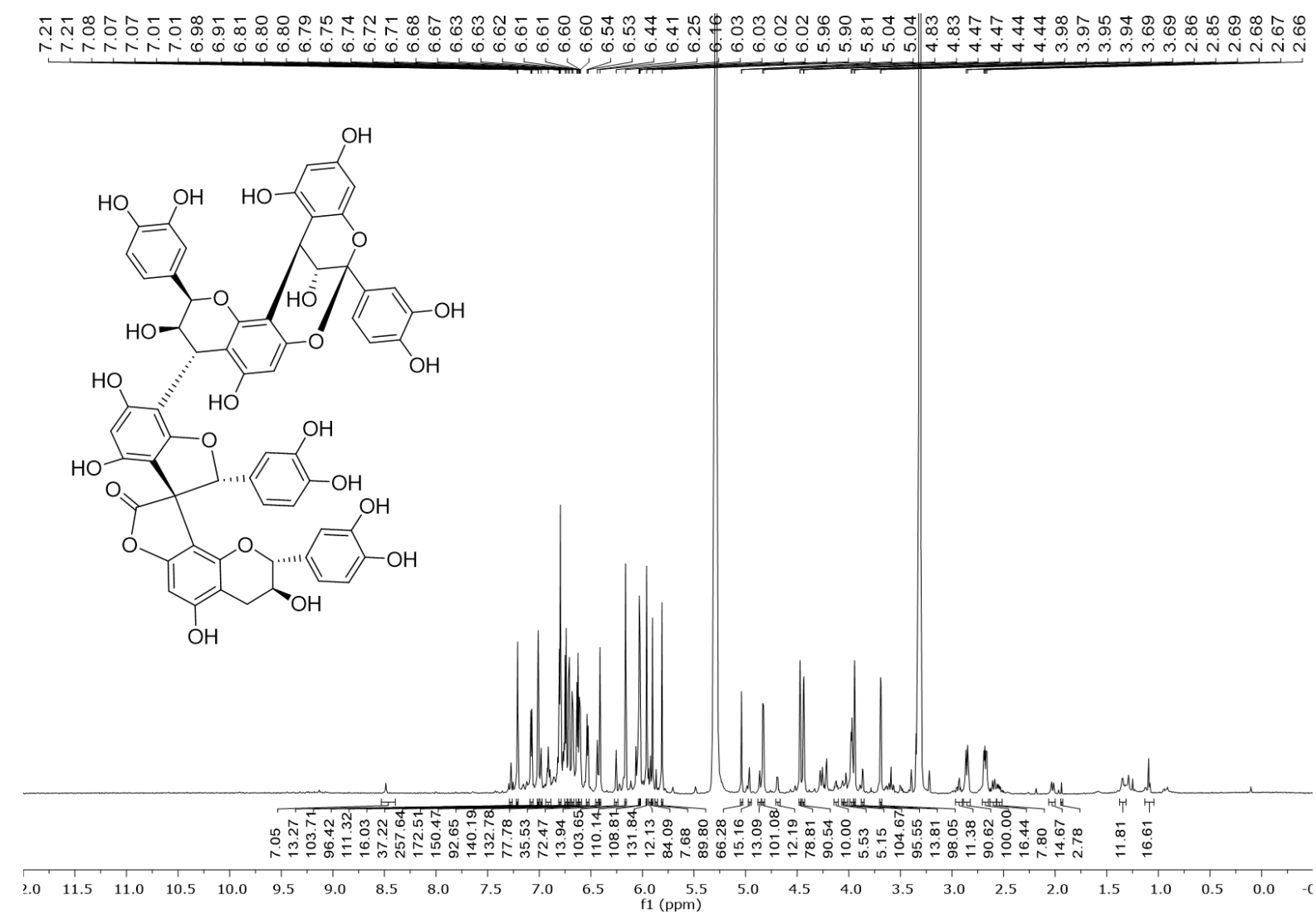

Quantitative ${ }^{1} \mathrm{H}$ NMR spectrum (800 MHz, $\mathrm{CD}_{3} \mathrm{OD}, 255 \mathrm{~K}$ )

\begin{tabular}{|l|r|}
\hline \multicolumn{2}{|c|}{ Compound 2 } \\
\hline Composition & Weight $\%$ \\
\hline Compound 2 & 86.7 \\
\hline Rotamer and/or analogues & 12.0 \\
\hline Formic acid & 0.1 \\
\hline Other impurities & 1.1 \\
\hline
\end{tabular}


Figure S27. Calculation of the purity of $\mathbf{3}$ by qHNMR using the 100\% method

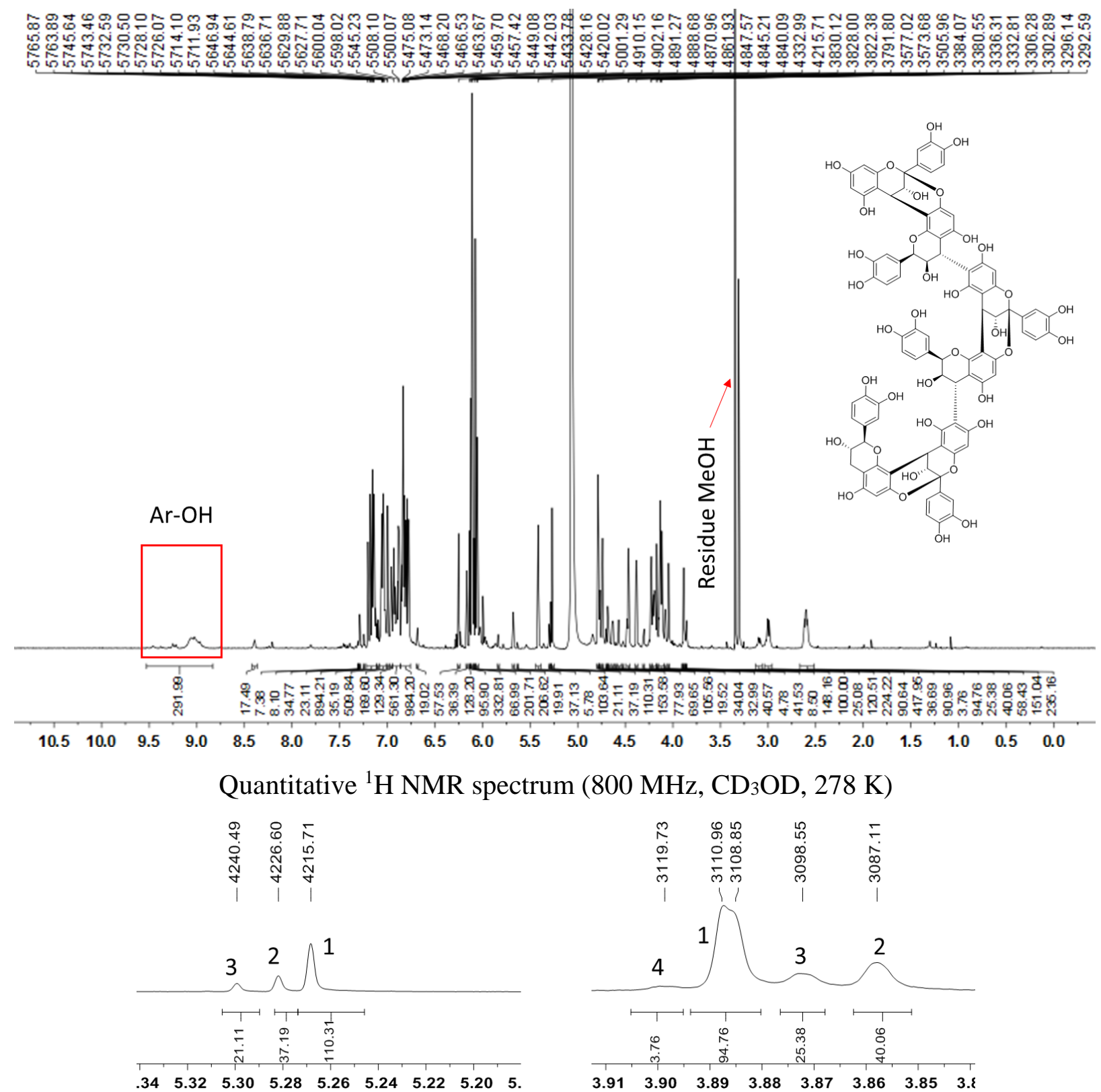

Three distinct rotamers can be observed

\begin{tabular}{|l|r|}
\hline \multicolumn{2}{|c|}{ Compound 3 } \\
\hline Composition & Weight $\%$ \\
\hline Rotamer 1 & 56.6 \\
\hline Rotamer 2 & 22.6 \\
\hline Rotamer 3 & 11.3 \\
\hline Other rotamers and/or analogues & 4.5 \\
\hline Methanol & 4.2 \\
\hline Formic acid & 0.3 \\
\hline Other impurities & 0.5 \\
\hline
\end{tabular}

